\title{
OPTIMAL INTERNAL DISSIPATION OF A DAMPED WAVE EQUATION USING A TOPOLOGICAL APPROACH
}

\author{
ARNAUD MÜNCH \\ Laboratoire de Mathématiques de Besançon \\ Université de Franche-Comté, UMR CNRS 6623, 16, route de Gray, 25030 Besançon, France \\ e-mail: arnaud.munch@univ-fcomte.fr
}

\begin{abstract}
We consider a linear damped wave equation defined on a two-dimensional domain $\Omega$, with a dissipative term localized in a subset $\omega$. We address the shape design problem which consists in optimizing the shape of $\omega$ in order to minimize the energy of the system at a given time $T$. By introducing an adjoint problem, we first obtain explicitly the (shape) derivative of the energy at time $T$ with respect to the variation in $\omega$. Expressed as a boundary integral on $\partial \omega$, this derivative is then used as an advection velocity in a Hamilton-Jacobi equation for shape changes. We use the level-set methodology on a fixed working Eulerian mesh as well as the notion of the topological derivative. We also consider optimization with respect to the value of the damping parameter. The numerical approximation is presented in detail and several numerical experiments are performed which relate the over-damping phenomenon to the well-posedness of the problem.
\end{abstract}

Keywords: shape design, wave equation, level set, topological derivative, numerical viscosity.

\section{Introduction and problem statement}

Let $T>0$ and $\Omega$ be a bounded domain of class $C^{2}\left(\mathbb{R}^{2}\right)$. We consider the standard damped wave equation on the cylinder,

$$
\left\{\begin{array}{l}
y_{\omega, a}^{\prime \prime}-\Delta y_{\omega, a}+a(\boldsymbol{x}) y_{\omega, a}^{\prime}=0 \quad \text { in } \Omega \times(0, T), \\
y_{\omega, a}=0 \quad \text { on } \partial \Omega \times(0, T), \\
y_{\omega, a}(\boldsymbol{x}, 0)=y_{0}(\boldsymbol{x}), \quad y_{\omega, a}^{\prime}(\boldsymbol{x}, 0)=y_{1}(\boldsymbol{x}) \quad \text { in } \Omega
\end{array}\right.
$$

where the prime denotes partial differentiation with respect to time. We assume that the damping potential $a \in L^{\infty}\left(\Omega, \mathbb{R}^{+}\right)$has the form

$$
a(\boldsymbol{x})=a \mathcal{X}_{\omega}(\boldsymbol{x}), \quad \forall \boldsymbol{x} \in \Omega
$$

for any $a \in \mathbb{R}^{+} . \mathcal{X}_{\omega}$ stands for the characteristic function of any domain $\omega$ strictly included in $\Omega$. Moreover, we assume that $\left(y_{0}, y_{1}\right) \in\left(H^{2}(\Omega) \cap H_{0}^{1}(\Omega)\right) \times H_{0}^{1}(\Omega)$ are independent of $\omega$ and $a$. The system (1) is well-posed (Lions and Magenes, 1968) and the unique solution satisfies

$$
\begin{gathered}
y_{\omega, a} \in C\left((0, T) ; H^{2}(\Omega) \cap H_{0}^{1}(\Omega)\right) \cap C^{1}\left((0, T) ; H_{0}^{1}(\Omega)\right) \\
\cap C^{2}\left((0, T) ; L^{2}(\Omega)\right) .
\end{gathered}
$$

For all $t>0$ the energy of the system (1),

$$
E(\omega, a, t)=\frac{1}{2} \int_{\Omega}\left(\left|y_{\omega, a}^{\prime}(\boldsymbol{x}, t)\right|^{2}+\left|\nabla y_{\omega, a}(\boldsymbol{x}, t)\right|^{2}\right) \mathrm{d} x,
$$

satisfies the following dissipation law:

$$
E^{\prime}(\omega, a, t)=-\int_{\Omega} a(\boldsymbol{x})\left|y_{\omega, a}^{\prime}(\boldsymbol{x}, t)\right|^{2} \mathrm{~d} x \leq 0,
$$

so that the linear wave equation (1) models the dissipation of a membrane by an internal actuator. Here $y_{\omega, a}(\boldsymbol{x}, t)$ denotes the transversal displacement at point $\boldsymbol{x}$ and time $t$, while $y_{0}$ and $y_{1}$ denote the initial position and velocity, respectively.

In this work, we consider the numerical solution of the following nonlinear problems:

$$
\begin{aligned}
& \left(P_{\omega}\right): \inf _{\omega \in \Omega} E(\omega, a, T), \\
& \left(P_{a}\right): \inf _{a \in L^{\infty}\left(\Omega, \mathbb{R}^{+}\right)} E(\omega, a, T) .
\end{aligned}
$$

Problem $\left(P_{\omega}\right)$, cf. Fig.1, is the so-called shape design problem and consists in optimizing the dissipation of the system with respect to $\omega$ at time $T$. It is a typical ill-posed problem in the sense that the infimum may not be reached in the class of the characteristic function; the optimal domain $\omega$ is then composed of an arbitrarily large number 
of disjoint components. In (Münch et al., 2006) (extended to the elasticity operator in (Münch et al., 2009)), a full well-posed relaxation of $\left(P_{\omega}\right)$ is given, using a non-convex variational analysis (we also refer to (Fahroo and Ito, 1997) for an analysis in the 1-D case). The analysis performed in (Münch et al., 2006) highlights the effect of the over-damping phenomenon characteristic to this damped wave equation (Freitas, 1999; LópezGómez, 1997), for which the dissipation vanishes for large values of the constant $a$. Precisely, $\left(P_{\omega}\right)$ looses its wellposedness as soon as this constant is large enough (with respect to the problem data). This observation motivates the numerical solution of Problem $\left(P_{a}\right)$, not studied so far in the general case (for the 1-D case, we refer to (Freitas, 1999)).

We highlight that in this work we do not make any geometrical assumption on $\omega$. This is in contrast to (Herbrard and Henrot, 2003; 2005), where the exponential decay rate of the energy was maximized with respect to $\omega$, assuming the optic geometrical condition, well-known in control theory (Bardos et al., 1992).

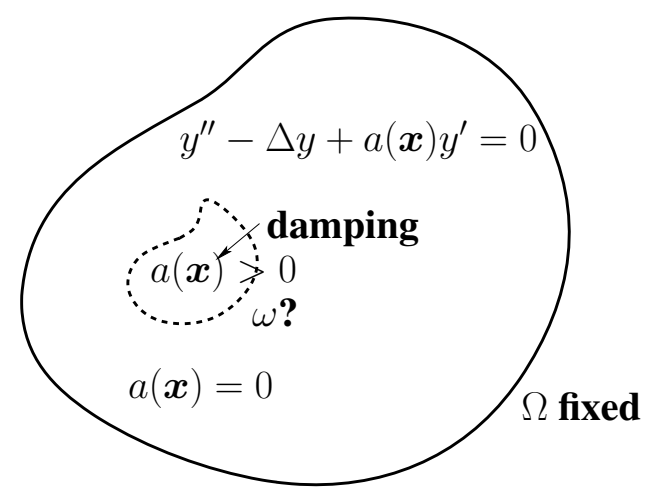

Fig. 1. Illustration of $\left(P_{\omega}\right)$ : Optimization of the location and shape of $\omega$, the support of the damping function $a$, in order to minimize the energy $E$ at time $T$.

This paper provides a numerical solution to the nonlinear problems (6) in order to complete the previous theoretical work (Münch et al., 2006). Given some additional geometrical conditions on $\omega$, observe that Problem $\left(P_{\omega}\right)$ is well posed (Henrot and Pierre, 2005). In order to use a gradient descent algorithm, a key point is to determine the derivative of $E$ with respect to $\omega$ and $a$. This is done using the domain derivative method (Delfour and Zolesio, 2001): the derivative with respect to $\omega$ is expressed as a curvilinear integral over $\Gamma=\partial \omega \times(0, T)$ and is a function of $y_{\omega, a}$ and $p_{\omega, a}$, the solution of an adjoint problem, and the jump of $a$ across $\partial \omega$. The derivative with respect to $a$, expressed in $\omega \times(0, T)$, is also a function of $p_{\omega, a}$. Then the optimal shape design problem $\left(P_{\omega}\right)$ is addressed with a level set approach, following recent works (Allaire et al., 2004; Wang et al., 2003); see also (Burger and Osher, 2005) for a survey regarding this question. From a numerical viewpoint, we discuss the approximation of the wave equation in such a way that the spurious high frequencies get damped out uniformly with respect to the discretization parameters. In order to ensure the convergence of the derivative of the energy derivative (which is necessary for the convergence of the discrete optimal design), we use a modified scheme with viscosity terms introduced and analyzed in (Münch and Pazoto, 2007).

The paper is organized as follows: The next section is devoted to the computation of the (shape) derivative of $E$ with respect to the variation of $\omega$ and also with respect to the damping function. Section 3 aims at recalling some aspects of level set methods and presents the minimization algorithm. Section 4 is devoted to the numerical approximation of the problem using a modified finite difference scheme. Section 5 presents several numerical simulations for Problems $\left(P_{\omega}\right),\left(P_{a}\right)$ and also for Problem $\left(P_{\omega, a}\right)$, which consists of minimizing the energy which respect to $\omega$ and $a$ simultaneously. We conclude with some remarks in Section 6.

\section{Existence results and the derivative of $E$}

2.1. Overview of existence results for $\left(P_{\omega}\right)$ and $\left(P_{a}\right)$. The aim of this part is to recall some assumptions which ensure the existence of at least one solution to Problems $\left(P_{\omega}\right)$ and $\left(P_{a}\right)$. Let us first make the following important remarks.

Remark 1. We underline that no condition is imposed on $\partial \omega$. This will allow us to use the level set approach, which consists in decoupling the description of the moving boundary $\partial \omega$ from that of the mesh of $\Omega$. It is also important to notice that, in the case where $a \in L^{\infty}(\Omega)$, the unique solution $y$ of (1) is such that $\nabla y$ is continuous on and through $\partial \omega$. Precisely, we recall that, if $\Omega=(\Omega \backslash \omega) \cup \omega \cup \partial \omega$, then

$$
\begin{aligned}
H^{1}(\Omega)= & \left\{v \in L^{2}(\Omega), v_{\mid \omega} \in H^{1}(\omega),\right. \\
& \left.v_{\mid(\Omega \backslash \omega)} \in H^{1}(\Omega \backslash \omega),[[v]]=0 \text { on } \partial \omega\right\},
\end{aligned}
$$

where $[[v]]$ denotes the jump in $v$ through $\partial \omega$. Then the interpretation of the following variational formulation associated with (1), for all $\varphi \in \mathcal{D}(\Omega)$ and for all $t>0$,

$$
\begin{aligned}
& \left\langle y_{\omega, a}^{\prime \prime}, \varphi\right\rangle_{\left(H^{-1}(\Omega), H_{0}^{1}(\Omega)\right)} \\
& \quad+\int_{\Omega} \nabla y_{\omega, a} \cdot \nabla \varphi \mathrm{d} x+\int_{\omega} a y_{\omega, a}^{\prime} \varphi d x=0
\end{aligned}
$$

implies, using the density of $\mathcal{D}(\Omega)$ in $H_{0}^{1}(\Omega)$, that $\int_{\partial \omega}\left[\left[\nabla y_{\omega, a} \cdot \nu\right]\right] \varphi \mathrm{d} \sigma=0$ for all $\varphi \in H^{1 / 2}(\partial \omega)$ and finally $\left[\left[\nabla y_{\omega, a} \cdot \boldsymbol{\nu}\right]\right]=0$. Then $\left[\left[y_{\omega, a}\right]\right]=0$ implies $\left[\left[\nabla y_{\omega, a} \cdot \boldsymbol{\tau}\right]\right]=0$.

Remark 2. The energy $E$ at time $T$ (in the sequel it is called the cost function) is not monotonous with re- 
spect to the area of $\omega$. Moreover, without any restriction on the area of $\omega$, one may conjecture that the trivial solution is $\omega=\Omega$. Similarly, one may conjecture that the inclusion $\omega_{1} \subset \omega_{2} \subset \Omega$ implies the inequality $E\left(\omega_{2}, a, T\right) \leq E\left(\omega_{1}, a, T\right), \forall T>0$. These two points, which seem open from the theoretical viewpoint, are numerically observed. Consequently, in the sequel we use the following subset:

$$
V_{L}=\{\omega \subset \Omega,|\omega|=L|\Omega|\}, \quad L \in(0,1),
$$

where $|\omega|$ denotes the measure of $\omega$, and replace $\left(P_{\omega}\right)$ by Problem $\left(P_{\omega, L}\right): \inf _{\omega \in V_{L}} E(\omega, a, T)$ for $L$ fixed in $(0,1)$.

It is well known that the static version of $\left(P_{\omega}\right)$ (and, a fortiori, $\left(P_{\omega}\right)$ ) is not well posed on the set of admissible shapes $V_{L}$ and usually has no solution (Delfour and Zolesio, 2001; Münch et al., 2006). In order to guarantee the existence of solutions, some geometrical constraints are required (Henrot and Pierre, 2005). We mention, e.g., the perimeter constraint leading to a well-posed problem. Since the domain $\omega$ is assumed time independent, it is easy to adapt the results of the static case. Using the independence of the initial condition $\left(y_{0}, y_{1}\right)$ with respect to $\omega$ and the decay of the energy, we obtain the following result (we refer to (Maestre et al., 2007) for a study of the time-dependent case).

Proposition 1. Let $L \in(0,1)$. We assume that $\omega$ is of class $C^{1,1}(\Omega)$ (uniformly Lipschitz continuous on $\Omega$ ). Then there exists at least one $\omega \subset V_{L}$ minimizing the functional $\omega \rightarrow E(\omega, a, T)$.

Since this work is devoted to numerical simulations, we do not reproduce the proof here and refer to (Münch et al., 2006) for a mathematical analysis. We also state without proof the following simpler result obtained using the independence of the initial condition with respect to the damping function $a$.

Proposition 2. Let $L \in(0,1)$. Let $\omega$ be fixed in $V_{L}$ and $a(\boldsymbol{x})=a \mathcal{X}_{\omega}(\boldsymbol{x})$. Then there exists at least one scalar $a \in \mathbb{R}_{\star}^{+}$minimizing the functional $a \rightarrow E(\omega, a, T)$.

2.2. Shape derivative of the cost with respect to $\boldsymbol{\omega}$. From now, we simply write $y$ for $y_{\omega, a}$. A standard procedure for this constrained problem $\left(P_{\omega, L}\right)$ is to relax the condition $\omega \in V_{L}$ via a penalization parameter $\varepsilon$ leading to the new problem:

$$
\left(P_{\omega, L}^{\varepsilon}\right): \inf _{\omega \subset \Omega} E^{\varepsilon}(\omega, a, T),
$$

where

$$
E^{\varepsilon}(\omega, a, T)=E(\omega, a, T)+\frac{1}{2} \varepsilon^{-1}(|\omega|-L|\Omega|)^{2} .
$$

In order to solve $\left(P_{\omega, L}^{\varepsilon}\right)$ using a gradient descent procedure, we now compute explicitly an expression of the derivative of the functional $E^{\varepsilon}$ with respect to $\omega$. The domain $\Omega$ is fixed and it is worth noticing that the initial condition $\left(y_{0}, y_{1}\right)$ is independent of $\omega$. Let $\eta \in \mathbb{R}^{+}$. From now on, we assume that $\omega$ is in $C^{1,1}(\Omega)$ and introduce a vector field $\boldsymbol{\theta} \in W^{1, \infty}\left(\Omega, \mathbb{R}^{2}\right)$, with $\boldsymbol{\theta}_{\mid \partial \Omega}=0$ and $\boldsymbol{\theta}$ not vanishing in a neighborhood of $\partial \omega$.

Definition 1. The derivative of the functional $E^{\varepsilon}$ with respect to a variation of $\omega \subset \Omega$ in the direction $\theta$ is defined as the Fréchet derivative in $W^{1, \infty}\left(\Omega, \mathbb{R}^{2}\right)$ at 0 of the map$\operatorname{ping} \boldsymbol{\theta} \rightarrow E^{\varepsilon}((I d+\eta \boldsymbol{\theta})(\omega))$, i.e.,

$$
\begin{aligned}
E^{\varepsilon}((I d & +\eta \boldsymbol{\theta})(\omega), a, T) \\
= & E^{\varepsilon}(\omega, a, T) \\
& \quad+\eta \frac{\partial E^{\varepsilon}(\omega, a, T)}{\partial \omega} \cdot \boldsymbol{\theta}+o\left(\|\boldsymbol{\theta}\|_{W^{1, \infty}\left(\Omega, \mathbb{R}^{2}\right)}\right) .
\end{aligned}
$$

We refer the reader to (Delfour and Zolesio, 2001; Henrot and Pierre, 2005) for more details. Moreover, the derivative is a continuous linear form on $W^{1, \infty}\left(\Omega, \mathbb{R}^{2}\right)$ and depends only of the field $\boldsymbol{\theta}$ in an arbitrary small neighborhood of $\partial \omega$.

Theorem 1. The derivative of $E^{\varepsilon}$ is given by the following expression:

$$
\begin{aligned}
\frac{\partial E^{\varepsilon}(\omega, a, T)}{\partial \omega} & \cdot \boldsymbol{\theta} \\
= & \int_{\partial \omega}\left[\varepsilon^{-1}(|\omega|-L|\Omega|)\right. \\
& \left.+a \int_{0}^{T} y^{\prime}(\boldsymbol{x}, t) p(\boldsymbol{x}, t) \mathrm{d} t\right] \boldsymbol{\theta} \cdot \boldsymbol{\nu} \mathrm{d} \sigma,
\end{aligned}
$$

where $\boldsymbol{\nu}$ denotes the outward normal and $p$ the solution of the following adjoint problem:

$$
\left\{\begin{array}{l}
p^{\prime \prime}-\Delta p-a(\boldsymbol{x}) p^{\prime}=0 \quad \text { in } \Omega \times(0, T), \\
p=0 \quad \text { on }(\partial \Omega \backslash \partial \omega) \times(0, T), \\
p(\boldsymbol{x}, T)=-y^{\prime}(\boldsymbol{x}, T) \quad \text { in } \Omega, \\
p^{\prime}(\boldsymbol{x}, T)=-a(\boldsymbol{x}) y^{\prime}(\boldsymbol{x}, T)-\Delta y(\boldsymbol{x}, T) \quad \text { in } \Omega .
\end{array}\right.
$$

Remark 3. If the function $a$ takes the value $a_{1}$ on $\omega$ and $a_{2}$ on $\Omega \backslash \omega$, the derivative takes the form

$$
\begin{aligned}
\frac{\partial E^{\varepsilon}(\omega, a, T)}{\partial \omega} & \boldsymbol{\theta} \\
= & \int_{\partial \omega}\left[\varepsilon^{-1}(|\omega|-L|\Omega|)\right. \\
& \left.+\int_{0}^{T}[[a]] y^{\prime}(\boldsymbol{x}, t) p(\boldsymbol{x}, t) \mathrm{d} t\right] \boldsymbol{\theta} \cdot \boldsymbol{\nu} \mathrm{d} \sigma,
\end{aligned}
$$

where the jump $[[a]]$ of $a$ across $\partial \omega$ is defined as $[[a]]=$ $a_{1}-a_{2}$. In particular, if the jump is equal to zero and if $\omega \subset V_{L}$, then the derivative with respect to $\omega$ is zero. 
Remark 4. From (3), $\left(p(T), p^{\prime}(T)\right) \in\left(H_{0}^{1}(\Omega), L^{2}(\Omega)\right)$. Therefore, the system (14) is well posed and there exists a unique solution $p$ such that

$$
p \in C\left((0, T) ; H_{0}^{1}(\Omega)\right) \cap C^{1}\left((0, T) ; L^{2}(\Omega)\right) .
$$

Consequently, $a y^{\prime} p \in C\left((0, T) ; W^{1,1}(\Omega)\right)$ and the derivative (1) is well defined as a function on $\partial \omega$.

Proof. In order to simplify the notation and to avoid duality products, we present a formal proof assuming that the solutions $y$ and $p$ are sufficiently regular to justify the integration by parts (we refer to (Cagnol and Zolésio, 1999) for rigorous developments). With the system (1) we associate a variational formulation keeping in mind that the domains $\Omega$ and $\omega$ are independent of time. For all $\varphi \in C\left((0, T) ; H_{0}^{1}(\Omega)\right)$, we then consider the formulation

$$
\left\{\begin{array}{r}
\int_{0}^{T} \int_{\Omega}\left(y^{\prime \prime} \varphi+\nabla y \cdot \nabla \varphi\right) \mathrm{d} x \mathrm{~d} t \\
\quad+\int_{0}^{T} \int_{\omega} a y^{\prime} \varphi \mathrm{d} x \mathrm{~d} t=0 \\
\int_{\Omega}\left(y(\cdot, 0)-y_{0}\right) \varphi(\cdot, 0) \mathrm{d} x=0 \\
\int_{\Omega}\left(y^{\prime}(\cdot, 0)-y_{1}\right) \varphi(\cdot, 0) \mathrm{d} x=0
\end{array}\right.
$$

and apply the derivation method in order to obtain the formulation associated with the first Lagrangian derivative $Y$. We obtain

$$
\left\{\begin{array}{l}
\int_{0}^{T} \int_{\Omega}\left(Y^{\prime \prime} \varphi+\nabla Y \cdot \nabla \varphi\right) \mathrm{d} x \mathrm{~d} t+\int_{0}^{T} \int_{\omega} a Y^{\prime} \varphi \mathrm{d} x \mathrm{~d} t \\
\quad+\int_{0}^{T} \int_{\Omega}\left(\mathcal{A}(\boldsymbol{\theta}) \cdot \nabla y \cdot \nabla \varphi+y^{\prime \prime} \operatorname{div} \boldsymbol{\theta} \varphi\right) \mathrm{d} x \mathrm{~d} t \\
\quad+\int_{0}^{T} \int_{\omega} a y^{\prime} \operatorname{div} \boldsymbol{\theta} \varphi \mathrm{d} x \mathrm{~d} t=0, \\
\int_{\Omega}\left(Y(\cdot, 0)-\nabla y_{0} \cdot \boldsymbol{\theta}\right) \varphi(\cdot, 0) \mathrm{d} x=0, \\
\int_{\Omega}\left(Y^{\prime}(\cdot, 0)-\nabla y_{1} \cdot \boldsymbol{\theta}\right) \varphi(\cdot, 0) \mathrm{d} x=0 .
\end{array}\right.
$$

The operator $\mathcal{A}: W^{1, \infty}\left(\Omega, \mathbb{R}^{2}\right) \rightarrow L^{\infty}\left(\Omega, \mathbb{R}^{2}\right)$ is defined as $\mathcal{A}(\boldsymbol{\theta})=\operatorname{div} \boldsymbol{\theta} I d-\left(\nabla \boldsymbol{\theta}+\nabla \boldsymbol{\theta}^{\star}\right)$, where $\nabla \boldsymbol{\theta}^{\star}$ signifies the transpose of $\nabla \boldsymbol{\theta}$. Using similar arguments, the derivative of the energy is

$$
\begin{aligned}
& \frac{\partial E(\omega, a, T)}{\partial \omega} \cdot \boldsymbol{\theta} \\
& =\frac{1}{2} \int_{\Omega}\left(\left(\left|y^{\prime}(T)\right|^{2}+|\nabla y(T)|^{2}\right) \operatorname{div} \boldsymbol{\theta}\right. \\
& \left.\quad-\left(\nabla \boldsymbol{\theta}+(\nabla \boldsymbol{\theta})^{\star}\right) \cdot \nabla y(T) \cdot \nabla y(T)\right) \mathrm{d} x \\
& \quad+\int_{\Omega}\left(y^{\prime}(T) Y^{\prime}(T)+\nabla y(T) \cdot \nabla Y(T)\right) \mathrm{d} x .
\end{aligned}
$$

Let us rewrite this derivative in terms of $y$ and $p$. A first computation leads to

$$
\begin{aligned}
\int_{\Omega}\left[y^{\prime}(T) Y^{\prime}(T)+\nabla y(T) \cdot \nabla Y(T)\right] \mathrm{d} x \\
=\int_{0}^{T} \int_{\Omega}\left(p^{\prime \prime} Y-Y^{\prime \prime} p\right) \mathrm{d} x \mathrm{~d} t \\
\quad+\int_{\Omega}\left(p^{\prime}(0) Y(0)-p(0) Y^{\prime}(0)\right) \mathrm{d} x \\
\quad+\int_{\omega} a y^{\prime}(T) Y(T) \mathrm{d} x .
\end{aligned}
$$

Using the formulation (1) and (14), we obtain

$$
\begin{aligned}
\int_{0}^{T} \int_{\Omega}( & \left.p^{\prime \prime} Y-Y^{\prime \prime} p\right) \mathrm{d} x \mathrm{~d} t \\
= & \int_{\omega} a(p(T) Y(T)-p(0) Y(0)) \mathrm{d} x \\
& +\int_{0}^{T} \int_{\Omega}\left(\mathcal{A}(\boldsymbol{\theta}) \cdot \nabla y \cdot \nabla p+y^{\prime \prime} p \operatorname{div} \boldsymbol{\theta}\right) \mathrm{d} x \mathrm{~d} t \\
& +\int_{0}^{T} \int_{\omega} a y^{\prime} p \operatorname{div} \boldsymbol{\theta} \mathrm{d} x \mathrm{~d} t .
\end{aligned}
$$

The relation $p(T)=-y^{\prime}(T)$ then implies

$$
\begin{aligned}
& \frac{\partial E(\omega, a, T)}{\partial \omega} \cdot \boldsymbol{\theta} \\
& =\frac{1}{2} \int_{\Omega}\left(\left(\left|y^{\prime}(T)\right|^{2}+|\nabla y(T)|^{2}\right) \operatorname{div} \boldsymbol{\theta}\right. \\
& \left.-\left(\nabla \boldsymbol{\theta}+(\nabla \boldsymbol{\theta})^{\star}\right) \cdot \nabla y(T) \cdot \nabla y(T)\right) \mathrm{d} x \\
& +\int_{0}^{T} \int_{\Omega}\left(\mathcal{A}(\boldsymbol{\theta}) \cdot \nabla y \cdot \nabla p+y^{\prime \prime} p \operatorname{div} \boldsymbol{\theta}\right) \mathrm{d} x \mathrm{~d} t \\
& +\int_{0}^{T} \int_{\omega} a y^{\prime} p \operatorname{div} \boldsymbol{\theta} \mathrm{d} x \mathrm{~d} t \\
& -\int_{\omega} a p(0) Y(0) \mathrm{d} x+\int_{\Omega}\left(p^{\prime}(0) Y(0)-p(0) Y^{\prime}(0)\right) \mathrm{d} x .
\end{aligned}
$$

Then, from $\mathcal{A}(\boldsymbol{\theta}) \cdot \nabla y \cdot \nabla p=\left(\theta_{1,1}-\theta_{2,2}\right)\left(y_{, 2} p_{, 2}-\right.$ $\left.y_{, 1} p_{, 1}\right)-\left(\theta_{1,2}+\theta_{2,1}\right)\left(y_{, 1} p_{, 2}+y_{, 2} p_{, 1}\right)$ and $\boldsymbol{\theta}_{\mid \partial \Omega}=0$, we obtain

$$
\begin{aligned}
\int_{0}^{T} \int_{\Omega}\left(\mathcal{A}(\boldsymbol{\theta}) \cdot \nabla y \cdot \nabla p+y^{\prime \prime} p \operatorname{div} \boldsymbol{\theta}\right) \mathrm{d} x \mathrm{~d} t \\
=\int_{0}^{T} \int_{\Omega}\left(\nabla p \cdot \boldsymbol{\theta}\left(\Delta y-y^{\prime \prime}\right)\right. \\
\left.\quad+\nabla y \cdot \boldsymbol{\theta} \Delta p-\nabla y^{\prime \prime} \cdot \boldsymbol{\theta} p\right) \mathrm{d} x \mathrm{~d} t \\
=\int_{0}^{T} \int_{\omega} \nabla p \cdot \boldsymbol{\theta} a y^{\prime} \mathrm{d} x \mathrm{~d} t+\int_{0}^{T} \int_{\Omega}(\nabla y \cdot \boldsymbol{\theta} \Delta p \\
\left.\quad-\nabla y^{\prime \prime} \cdot \boldsymbol{\theta} p\right) \mathrm{d} x \mathrm{~d} t .
\end{aligned}
$$


Additional integrations by part, using the fact that $\boldsymbol{\theta}$ is time-independent, lead to the following expression:

$$
\begin{aligned}
& \frac{\partial E(\omega, a, T)}{\partial \omega} \cdot \boldsymbol{\theta} \\
& =\frac{1}{2} \int_{\Omega}\left(\left(\left|y^{\prime}(T)\right|^{2}+|\nabla y(T)|^{2}\right) \operatorname{div} \boldsymbol{\theta}\right. \\
& \left.\quad-\left(\nabla \boldsymbol{\theta}+(\nabla \boldsymbol{\theta})^{\star}\right) \cdot \nabla y(T) \cdot \nabla y(T)\right) \mathrm{d} x \\
& \quad+\int_{0}^{T} \int_{\omega} \operatorname{div}\left(a y^{\prime} p \boldsymbol{\theta}\right) \mathrm{d} \omega \mathrm{d} t \\
& \quad+\int_{\Omega}\left(\nabla y^{\prime}(T) \cdot \boldsymbol{\theta} y^{\prime}(T)-\nabla y(T) \cdot \boldsymbol{\theta} \Delta y(T)\right) \mathrm{d} x .
\end{aligned}
$$

Then, from

$$
\operatorname{div}\left(\left|y^{\prime}(T)\right|^{2} \boldsymbol{\theta}\right)=\left|y^{\prime}(T)\right|^{2} \operatorname{div} \boldsymbol{\theta}+2 y^{\prime}(T) \nabla y^{\prime}(T) \cdot \boldsymbol{\theta}
$$

and the relation

$$
\begin{aligned}
& \int_{\Omega} \nabla\left(|\nabla y(T)|^{2}\right) \cdot \boldsymbol{\theta} \\
&=-\int_{\Omega}\left(\nabla \boldsymbol{\theta}+\nabla \boldsymbol{\theta}^{\star}\right) \cdot \nabla y(T) \cdot \nabla y(T) \\
&-2 \int_{\Omega} \nabla y(T) \cdot \boldsymbol{\theta} \Delta y(T),
\end{aligned}
$$

we finally obtain

$$
\begin{aligned}
\frac{\partial E(\omega, a, T)}{\partial \omega} \cdot \boldsymbol{\theta} \\
=\frac{1}{2} \int_{\Omega} \operatorname{div}\left(\left(\left|y^{\prime}(T)\right|^{2}+|\nabla y(T)|^{2}\right) \boldsymbol{\theta}\right) \mathrm{d} x \\
\quad+\int_{0}^{T} \int_{\omega} \operatorname{div}\left(a y^{\prime} p \boldsymbol{\theta}\right) \mathrm{d} x \mathrm{~d} t \\
=\int_{0}^{T} \int_{\partial \omega} a y^{\prime} p \boldsymbol{\theta} \cdot \boldsymbol{\nu} d \sigma \mathrm{d} t
\end{aligned}
$$

using $\boldsymbol{\theta}_{\mid \partial \Omega}=0$ in the first integral. Finally, from

$$
\frac{\partial}{\partial \omega}(|\omega|-L|\Omega|) \cdot \boldsymbol{\theta}=\int_{\partial \omega} \boldsymbol{\theta} \cdot \boldsymbol{\nu} \mathrm{d} \sigma
$$

we obtain the relation (1).

2.3. Derivative of the cost with respect to the coefficient $a$. We now assume that the domain $\omega$ is fixed in $\Omega$ and optimize $E$ with respect to the value of the damping coefficient $a$. Since the cost is not monotonous with respect to $a$, it is not necessary to introduce a penalization argument here. Let us consider a perturbation of $a$ :

$$
a^{\eta}(\boldsymbol{x})=a(\boldsymbol{x})+\eta a^{1}(\boldsymbol{x}), \quad \boldsymbol{x} \in \Omega,
$$

while assuming $\eta$ small enough for $a^{\eta}$ to remain in $L^{\infty}\left(\Omega, \mathbb{R}^{+}\right)$. We then assume that the variation of the solution of the wave equation can be written as follows: $y^{\eta}=y+\eta Y+O\left(\eta^{2}\right)$.
Theorem 2. The derivative of $E$ with respect to $a$ is

$$
\frac{\partial E(\omega, a, T)}{\partial a} \cdot a^{1}=\int_{0}^{T} \int_{\omega} a^{1} y^{\prime}(\boldsymbol{x}, t) p(\boldsymbol{x}, t) \mathrm{d} x \mathrm{~d} t,
$$

where $p$ is the solution of (14).

Proof. The proof is simpler than in the previous case. We obtain that $Y$ and $a^{1}$ solve

$$
\begin{aligned}
\int_{0}^{T} \int_{\Omega} & \left(Y^{\prime \prime} \varphi+\nabla Y \cdot \nabla \varphi\right) \mathrm{d} x \mathrm{~d} t \\
& +\int_{0}^{T} \int_{\omega}\left(a Y^{\prime}+a^{1} y^{\prime}\right) \varphi \mathrm{d} x \mathrm{~d} t=0
\end{aligned}
$$

for all $\varphi \in C\left((0, T) ; H_{0}^{1}(\Omega)\right)$. Furthermore, since the initial conditions are independent of $a$, we have $Y(\cdot, 0)=$ 0 and $Y^{\prime}(\cdot, 0)=0$ in $\Omega$. The derivative of $E$ with respect to $a$ is then

$$
\begin{aligned}
& \frac{\partial E^{\varepsilon}(\omega, a, T)}{\partial a} \cdot a^{1} \\
& \quad=\frac{\partial E(\omega, a, T)}{\partial a} \cdot a^{1} \\
& \quad=\int_{\Omega}\left(y^{\prime}(T) Y^{\prime}(T)+\nabla y(T) \cdot \nabla Y(T)\right) \mathrm{d} x .
\end{aligned}
$$

We then adapt (20) to obtain

$$
\begin{aligned}
\int_{\Omega} & {\left[y^{\prime}(T) Y^{\prime}(T)+\nabla y(T) \cdot \nabla Y(T)\right] \mathrm{d} x } \\
= & \int_{0}^{T} \int_{\Omega}\left(p^{\prime \prime} Y-Y^{\prime \prime} p\right) \mathrm{d} x \mathrm{~d} d t+\int_{\omega} a y^{\prime}(T) Y(T) \mathrm{d} x .
\end{aligned}
$$

$Y$ being solution of (30), we obtain

$$
\begin{aligned}
& \int_{0}^{T} \int_{\Omega}\left(p^{\prime \prime} Y-Y^{\prime \prime} p\right) \mathrm{d} x \mathrm{~d} t \\
& =\int_{\omega} a(Y(T) p(T)-Y(0) p(0)) \mathrm{d} x+\int_{0}^{T} \int_{\omega} a^{1} y^{\prime} p \mathrm{~d} x \mathrm{~d} t \\
& =-\int_{\omega} a Y(T) y^{\prime}(T) \mathrm{d} x+\int_{0}^{T} \int_{\omega} a^{1} y^{\prime} p \mathrm{~d} x \mathrm{~d} t
\end{aligned}
$$

and finally the relation 29 .

2.4. Topological derivative. In a similar manner, we may also compute the topological derivative associated with $E$, the notion introduced in (Sokołowski and Żochowski, 1999) and then used efficiently in the context of shape optimization (see, e.g., (Allaire et al., 2005; Fulmanski et al., 2008)).

Theorem 3. For any $\boldsymbol{x}_{\mathbf{0}} \in \Omega$ and $\rho$ such that $D\left(x_{0}, \rho\right) \equiv$ $\left\{\boldsymbol{x} \in \mathbb{R}^{2}, \operatorname{dist}\left(\boldsymbol{x}, \boldsymbol{x}_{\mathbf{0}}\right) \leq \rho\right\} \subset \Omega$, the functional associ- 
ated with $D\left(\boldsymbol{x}_{\mathbf{0}}, \rho\right)$ may be expressed as

$$
\begin{aligned}
& E\left(D\left(\boldsymbol{x}_{\mathbf{0}}, \rho\right), a, T\right) \\
& \quad=E(\emptyset, a, T) \\
& \quad+\pi \rho^{2} \int_{0}^{T} a y_{\emptyset, 0}^{\prime}\left(\boldsymbol{x}_{\mathbf{0}}, t\right) p_{\emptyset, 0}\left(\boldsymbol{x}_{\mathbf{0}}, t\right) \mathrm{d} t+o\left(\rho^{2}\right)
\end{aligned}
$$

only in terms of the conservative solutions $y_{\emptyset, 0}$ and $p_{\emptyset, 0}$. The factor of $\rho^{2}$ is called the topological derivative of $E$.

Proof. The computation is very similar to the computation of the shape derivative (we refer the reader to (Sokołowski and Żochowski, 1999) for general developments). In our simple situation, we may obtain a relation similar to (34) by using the interplay between $\omega$ and $a$ (i.e., $E(\omega, 0, T)=E(\emptyset, a, T)$ for all $a$ and $\omega)$ and the variation of $E$ with respect to $a$. Precisely, taking $a=0$ and $\omega=D\left(\boldsymbol{x}_{\mathbf{0}}, \rho\right) \subset \Omega$ in the equality

$$
\begin{aligned}
& E\left(\omega, a+\eta a^{1}, T\right) \\
& \quad=E(\omega, a, T) \\
& \quad+\eta a^{1} \int_{\omega} \int_{0}^{T} y_{\omega, a}^{\prime}(\boldsymbol{x}, t) p_{\omega, a}(\boldsymbol{x}, t) \mathrm{d} t \mathrm{~d} x+o(\eta)
\end{aligned}
$$

leads to

$$
\begin{aligned}
& E\left(D\left(\boldsymbol{x}_{\mathbf{0}}, \rho\right), \eta a^{1}, T\right) \\
& =E\left(D\left(\boldsymbol{x}_{\mathbf{0}}, \rho\right), 0, T\right) \\
& \quad+\eta a^{1} \int_{D\left(\boldsymbol{x}_{\mathbf{0}}, \rho\right)} \int_{0}^{T} y_{\emptyset, 0}^{\prime}(\boldsymbol{x}, t) p_{\emptyset, 0}(\boldsymbol{x}, t) \mathrm{d} t \mathrm{~d} x+o(\eta) .
\end{aligned}
$$

Then, from $E\left(D\left(\boldsymbol{x}_{\mathbf{0}}, \rho\right), 0, T\right)=E\left(\emptyset, \eta a^{1}, T\right)$ (and replacing $\eta a^{1}$ by $a$ ), we obtain the difference of the energies associated with the dissipative and conservative cases, respectively, as a function of only the solutions of the conservative case $y_{\emptyset, 0}$ and $p_{\emptyset, 0}$,

$$
\begin{aligned}
& E\left(D\left(\boldsymbol{x}_{\mathbf{0}}, \rho\right), a, T\right) \\
& =E(\emptyset, a, T) \\
& \quad+a \int_{D\left(\boldsymbol{x}_{\mathbf{0}}, \rho\right)} \int_{0}^{T} y_{\emptyset, 0}^{\prime}(\boldsymbol{x}, t) p_{\emptyset, 0}(\boldsymbol{x}, t) \mathrm{d} t \mathrm{~d} x+o(a) .
\end{aligned}
$$

We then easily get (34). Once again, this relation highlights the balance between $a$ and $\omega$ (or, equivalently, $\rho$ ) and illustrates the over-damping phenomenon. We will use these relation to obtain an efficient prediction of $\omega$, for $a$ or $\rho$ small enough (see Section 5).

\section{Minimization of the cost}

Thanks to the previous computations, we are now in a position to apply a gradient descent method to the minimization of the objective function $E^{\varepsilon}$ with respect to the position of $\omega$ and for $E$ with respect to the value of the function $a$, respectively.

3.1. Minimization of $E^{\varepsilon}$ with respect to $\omega$-Level set approach. From (1), the shape derivative is

$$
\frac{\partial E^{\varepsilon}(\omega, a, T)}{\partial \omega} \cdot \boldsymbol{\theta}=\int_{\partial \omega} j^{\varepsilon}\left(y_{\omega, a}, p_{\omega, a}, T\right) \boldsymbol{\theta} \cdot \boldsymbol{\nu} \mathrm{d} \sigma
$$

with

$$
\begin{aligned}
j^{\varepsilon} & \left(y_{\omega, a}, p_{\omega, a}, T\right) \\
\quad & =\varepsilon^{-1}(|\omega|-L|\Omega|)+a \int_{0}^{T} y_{\omega, a}^{\prime}(\boldsymbol{x}, t) p_{\omega, a}(\boldsymbol{x}, t) \mathrm{d} t
\end{aligned}
$$

defined on $\Omega$. A descent direction is found by defining on $\partial \omega$ the vector field $\boldsymbol{\theta}$ as follows:

$$
\boldsymbol{\theta}=-j^{\varepsilon}\left(y_{\omega}, p_{\omega}, T\right) \boldsymbol{\nu}
$$

We then update the shape $\omega$ as $\omega^{\eta}=(I d+\eta \boldsymbol{\theta})(\omega)$ (we recall that $\omega$ is in $C^{1,1}(\Omega)$ ). The parameter $\eta>0$ denotes a descent step small enough so that the formal relation

$$
\begin{aligned}
& E^{\varepsilon}\left(\omega^{\eta}, a, T\right) \\
& \quad=E^{\varepsilon}(\omega, a, T) \\
& \quad-\eta \int_{\partial \omega}\left(j^{\varepsilon}\left(y_{\omega, a}, p_{\omega, a}, T\right)\right)^{2} \mathrm{~d} \sigma+O\left(\eta^{2}\right)
\end{aligned}
$$

guarantees a decrease in $E^{\varepsilon}$. This method can be implemented in the Lagrangian framework: it suffices to form meshes in the domains $\omega, \Omega \backslash \omega$ and then advect the mesh according to the descent direction $\boldsymbol{\theta}$ defined on $\partial \omega$ by (39). This imposes meshing the moving interface $\partial \omega$. Morever, the re-meshing of the domain at each step may produce a costly method. Finally, the change in the topology of $\partial \omega$ is quite difficult to handle with this approach. Therefore, following recent works in optimal shape design (Allaire et al., 2004; Wang et al., 2003), we adopt an Eulerian approach and use a level-set method to capture the shape $\omega$ on a fixed mesh. Let us briefly recall the main features of this method.

The level set approach introduced in (Osher and Sethian, 1988) (see Osher and Fedkiw, 2002; 1996) for a survey) consists in giving a description of the evolving interface $\partial \omega$ independent of the discretizing mesh on $\Omega$. We define the level-set function $\psi$ in $\Omega$ in such a way that

$$
\begin{cases}\psi(\boldsymbol{x}) \leq 0 & \boldsymbol{x} \in \omega, \\ \psi(\boldsymbol{x})=0 & \boldsymbol{x} \in \partial \omega, \\ \psi(\boldsymbol{x}) \geq 0 & \boldsymbol{x} \in \Omega \backslash \omega .\end{cases}
$$

Therefore, the evolving interface $\partial \omega$ is characterized by

$$
\partial \omega=\{\boldsymbol{x}(\tau) \in \Omega \text { such that } \psi(\boldsymbol{x}(\tau), \tau)=0\},
$$


where $\tau$ denotes a pseudo-time variable, increasing with time, which may be the real time, a load or in our case, the iterations of a given algorithm. The differentiation of (42) with respect to $\tau$ then leads to

$$
\frac{\partial \psi}{\partial \tau}(\boldsymbol{x}(\tau), \tau)+\nabla \psi(\boldsymbol{x}(\tau), \tau) \cdot \frac{\mathrm{d} \boldsymbol{x}(\tau)}{\mathrm{d} \tau}=0
$$

Denoting by $\boldsymbol{F}$ the speed in the outward normal direction, such that $\frac{\mathrm{d} \boldsymbol{x}(\tau)}{\mathrm{d} \tau} \cdot \boldsymbol{\nu}=\boldsymbol{F}(\boldsymbol{x}(\tau))$, where $\boldsymbol{\nu}=$ $\nabla \psi /|\nabla \psi|$, we obtain the following nonlinear HamiltonJacobi equation of the first order for $\psi$ :

$$
\begin{aligned}
\frac{\partial \psi}{\partial \tau}(\boldsymbol{x}, \tau)+F(\boldsymbol{x}, \tau)|\nabla \psi(\boldsymbol{x}, \tau)| & =0 \\
\text { given } & \psi(\boldsymbol{x}, \tau=0) .
\end{aligned}
$$

Assuming that the shape $\partial \omega$ evolves in pseudo-time $\tau$ with the normal velocity $\boldsymbol{F}=-j^{\varepsilon}\left(y_{\omega, a}, p_{\omega, a}, T\right) \boldsymbol{\nu}$ as proposed in (39), the transport of the level set function $\psi$ is therefore equivalent to moving the boundary of $\omega$ (the zero level-set of $\psi$ ) along the descent gradient direction $-\partial E^{\varepsilon} / \partial \omega$. Consequently, the partial differential system which has to be solved is given by

$$
\left\{\begin{array}{l}
\frac{\partial \psi}{\partial \tau}-j^{\varepsilon}\left(y_{\omega, a}, p_{\omega, a}, T\right)|\nabla \psi|=0 \quad \text { in } \Omega \times(0, \infty), \\
\psi(\cdot, \tau=0)=\psi_{0} \quad \text { in } \Omega \\
\psi=\psi_{0}>0 \quad \text { on } \partial \Omega \times(0, \infty) .
\end{array}\right.
$$

We further impose that $\psi$ should be constant and positive on $\Omega$ in order to ensure that $\partial \omega \cap \partial \Omega=\emptyset$. Finally, because of its advection, the level-set function may become too flat or too steep yielding either large errors in the location of its zero level or large errors in the evaluation of its gradient by finite differences. Therefore, a standard trick (see (Osher and Fedkiw, 1996)) consists in replacing the level-set $\psi$ at the pseudo time $\tau_{0}$ by the regularized one, which solves the following problem:

$$
\left\{\begin{array}{l}
\frac{\partial \tilde{\psi}}{\partial \tau}+\operatorname{sign}\left(\psi\left(\tau_{0}\right)\right)(|\nabla \tilde{\psi}|-1)=0 \quad \text { in } \Omega \times(0, \infty), \\
\tilde{\psi}(\cdot, \tau=0)=\psi\left(\tau_{0}\right) \quad \text { in } \Omega
\end{array}\right.
$$

admitting as a stationary solution the signed distance to the initial interface $\left\{\psi\left(\boldsymbol{x}, \tau_{0}\right)=0\right\}$.

3.2. Minimization of $\boldsymbol{E}$ with respect to $\boldsymbol{a}$. Similarly, we use the expression (29) to minimize the cost function with respect to $a$. The quantity $a$ being constant on $\omega$, a descent direction is obtained by defining $a^{1}$ as follows:

$$
a^{1}=-\int_{0}^{T} \int_{\omega} y^{\prime}(\boldsymbol{x}, t) p(\boldsymbol{x}, t) \mathrm{d} x \mathrm{~d} t .
$$

Then, we update $a$ on $\omega$ as $a^{\eta}=a+\eta a^{1}$ on $\omega$, where $\eta>0$ signifies a descent step small enough so that

$$
\begin{aligned}
& E\left(\omega, a^{\eta}, T\right) \\
& =E(\omega, a, T) \\
& \quad-\eta\left(\int_{0}^{T} \int_{\omega} y^{\prime}(\boldsymbol{x}, t) p(\boldsymbol{x}, t) \mathrm{d} x \mathrm{~d} t\right)^{2}+O\left(\eta^{2}\right)
\end{aligned}
$$

guarantees a decrease in $E$.

Remark 5. If we assume that $a$ may vary in $\omega$, then the descent direction is

$$
a^{1}(\boldsymbol{x})=-\int_{0}^{T} y^{\prime}(\boldsymbol{x}, t) p(\boldsymbol{x}, t) \mathrm{d} t
$$

leading to

$$
\frac{\partial E(\omega, a, T)}{\partial a} \cdot a^{1}=-\int_{\omega}\left(\int_{0}^{T} y^{\prime}(\boldsymbol{x}, t) p(\boldsymbol{x}, t) \mathrm{d} t\right)^{2} \mathrm{~d} x .
$$

\section{Numerical approximation and optimization algorithm}

In this section we present some important numerical aspects. In particular, we focus on the importance of adding some viscosity terms in the usual discrete approximation of the wave systems (1) and (14), which are more sensitive to numerical approximation than elliptic or parabolic systems.

4.1. Solution of the wave equations (1) and (14)Introduction of viscosity terms. For simplicity, we take $\Omega=(0,1)^{2}$ and choose to approximate the wave system with finite difference schemes. Let us consider $J \in$ $\mathbb{N}, h=1 /(J+1)$ and a uniform grid $\left(x_{1, i}, x_{2, j}\right)_{(i, j)}$ of $\Omega$ such that $0=x_{k, 0}<x_{k, 1}<\cdots<x_{k, J}<x_{k, J+1}=1$, with $x_{k, j}=j h$ and $k=1,2$. Let us also consider $N \in \mathbb{N}$, $\Delta t=T / N$ and a uniform grid of the time interval $(0, T)$ given by $0=t_{0}<t_{1}<\cdots<t_{N}=T$, with $t_{n}=n \Delta t$. Here $h$ and $\Delta t$ denote the space and time step, respectively. Let us denote by $y_{i, j}^{n}$ the approximation of $y$ at the point $\left(x_{1, i}, x_{2, j}\right)$ and time $t_{n}$ :

$$
\begin{aligned}
& y_{i, j}^{n} \approx y\left(x_{1, i}, x_{2, j}, t_{n}\right), \\
& 0 \leq i, j \leq J+1, \quad 0 \leq n \leq N .
\end{aligned}
$$

The simplest way (Cohen, 2002) to approximate the wave equation is to approximate the derivative in time by a centered finite difference as follows:

$$
\begin{aligned}
& 2 \Delta t y^{\prime}=y^{n+1}-y^{n-1}+O\left(\Delta t^{3}\right), \\
& \Delta t^{2} y^{\prime \prime}=y^{n+1}-2 y^{n}+y^{n-1}+O\left(\Delta t^{4}\right),
\end{aligned}
$$


and to approximate the Laplacian by the five-point finite difference

$$
\begin{aligned}
h^{2} \Delta y\left(x_{1, i}, x_{2, j}\right)= & y_{i+1, j}+y_{i-1, j}+y_{i, j+1} \\
& +y_{i, j-1}-4 y_{i, j}+O\left(h^{4}\right) \\
\equiv & h^{2} \Delta_{h} y_{i, j}+O\left(h^{4}\right)
\end{aligned}
$$

leading to a centered scheme of order two in space and time, which is stable under the condition $\Delta t \leq h / \sqrt{2}$ (we refer to (Münch and Pazoto, 2007) for details). However, as observed initially in (Banks et al., 1991) in the context of stabilization and in (Glowinski et al., 1989) in the context of exact controllability, this scheme is not uniformly convergent with respect to the dissipation property.

The interaction of waves with a numerical mesh produces dispersion phenomena and spurious high frequencies. Because of this nonphysical interaction of waves with the discrete medium, the velocity of propagation of numerical waves may converge to zero when the wavelength of solutions is of the order of the size of the mesh. Consequently, the time needed to uniformly damp the numerical waves from a subset of $\Omega \backslash \omega$ in which they propagate may tend to infinity as the mesh becomes finer. Thus, the dissipation mechanism may disappear completely if the initial conditions are represented by high frequency components (see (Münch and Pazoto, 2007) for numerical illustrations). This is the case for discontinuous initial data. Mathematically speaking, the convergence of the solution in the energy norm is not ensured.

We replace the standard scheme by the scheme associated with the following equation:

$$
y^{\prime \prime}-\Delta y+a(\boldsymbol{x}) y^{\prime}-h^{2} \Delta y^{\prime}=0 \quad \text { in } \Omega \times(0, T)
$$

including the so-called viscous term $h^{2} \Delta y^{\prime}$ negligible for the low frequency components and of the order of the energy for the high frequency ones. The modified implicit scheme is then

$$
\left\{\begin{array}{c}
\left(1+\frac{\Delta t}{2}\left(a_{i, j}-\Delta_{h}\right)\right) y_{i, j}^{n+1} \\
=\left(2+r^{2} \Delta_{h}\right) y_{i, j}^{n} \\
\quad-\left(1-\frac{\Delta t}{2}\left(a_{i, j}-\Delta_{h}\right)\right) y_{i, j}^{n-1}, \\
\quad 1 \leq i, j \leq J, \quad 1 \leq n \leq N, \\
y_{i, j}^{n}=0, i=\{0, J+1\} \\
\quad \text { or } j=\{0, J+1\}, 1 \leq n \leq N, \\
y_{i, j}^{0}=y_{0}\left(x_{1, i}, x_{2, j}\right), \quad \\
y_{i, j}^{1}=\left(1+\frac{r^{2}}{2} \Delta_{h}\right) y_{i, j}^{0}+\Delta t y_{1}\left(x_{1, i}, x_{2, j}\right), \\
1 \leq i, j \leq J,
\end{array}\right.
$$

where $r=\Delta t / h$. In (Münch and Pazoto, 2007), it is shown that this scheme is a convergent approximation of
(1) under the condition $\Delta t \leq h / \sqrt{2}$ and provides a uniform approximation of the energy as $h$ and $\Delta t$ tend to zero. The same modification is needed for the adjoint problem. This is because the initial conditions at time $T$ defined by $p(T)=-y^{\prime}(T)$ and $p^{\prime}(T)=a(\boldsymbol{x}) p(T)-$ $\Delta y(T)$ are a priori less regular than $y_{0}$ and $y_{1}$. Therefore, we consider the discretization of the following equation:

$$
p^{\prime \prime}-\Delta p-a(\boldsymbol{x}) p^{\prime}+h^{2} \Delta p^{\prime}=0 \text { in } \Omega \times(0, T)
$$

leading to

$$
\left\{\begin{array}{c}
\left(1+\frac{\Delta t}{2}\left(a_{i, j}-\Delta_{h}\right)\right) p_{i, j}^{n-1} \\
=\left(2+r^{2} \Delta_{h}\right) p_{i, j}^{n} \\
\quad-\left(1-\frac{\Delta t}{2}\left(a_{i, j}-\Delta_{h}\right)\right) p_{i, j}^{n+1}, \\
\quad 1 \leq i, j \leq J, \quad 1 \leq n \leq N, \\
p_{i, j}^{n}=0, i=\{0, J+1\} \\
\text { or } j=\{0, J+1\}, 1 \leq n \leq N, \\
p_{i, j}^{N}=-\frac{y_{i, j}^{N+1}-y_{i, j}^{N-1}}{2 \Delta t}, \\
p_{i, j}^{N-1}=\left(1-a_{i, j} \Delta t\right) p_{i, j}^{N}+\frac{\Delta t}{h^{2}} \Delta_{h} y_{i, j}^{N}, \\
\quad 1 \leq i, j \leq J .
\end{array}\right.
$$

4.2. Solution of the Hamilton-Jacobi equation. Let us now consider the solution of the non-linear HamiltonJacobi equation (45). We introduce a parameter $\Delta \tau>0$ and denote by $\psi_{i, j}^{k}$ the approximation of the function $\psi$ at the point $x_{i, j}=\left(x_{1, i}, x_{2, j}\right)$ and at the pseudotime $\tau=k \Delta t$. We note by $j_{i, j}^{\varepsilon}$ the approximation of $j^{\varepsilon}\left(y_{\omega, a}\left(x_{i, j}\right), p_{\omega, a}\left(x_{i, j}\right)\right)$ such that

$$
\begin{aligned}
j_{i, j}^{\varepsilon}= & \varepsilon^{-1}\left(\left|\omega_{h}\right|-L\left|\Omega_{h}\right|\right) \\
& +a_{i, j} \sum_{n=0}^{N} \frac{y_{i, j}^{n+1}-y_{i, j}^{n-1}}{2} p_{i, j}^{n},
\end{aligned}
$$

where we let $a_{i, j}=a\left(x_{i, j}\right)=a \mathcal{X}_{\left(\psi_{i, j}<0\right)},\left|\Omega_{h}\right|=1$ and $\left|\omega_{h}\right|=\left\|\mathcal{X}_{\left(\psi_{h}\left(x_{i, j}\right) \leq 0\right)}\right\|_{L_{h}^{1}(\Omega)}$. The hyperbolic system (45) is solved using an explicit weighted essentially nonoscillatory scheme of order one in pseudo-time $\tau$ and of order two in space (see (Osher and Fedkiw, 2002; 1996) for a complete description):

$$
\begin{aligned}
\frac{\psi^{k+1}-\psi^{k}}{\Delta \tau} & +\left(\max \left(-j^{\varepsilon}\left(y^{k}, p^{k}\right), 0\right) \nabla_{k}^{+}\right. \\
& \left.+\min \left(-j^{\varepsilon}\left(y^{k}, p^{k}\right), 0\right) \nabla_{k}^{-}\right)=0
\end{aligned}
$$

for all $k>0$, where $\left(\nabla_{k}^{+}, \nabla_{k}^{-}\right)$stands for forward and upward approximations of $\left|\nabla \psi^{k}\right|$. This explicit scheme 
is stable under the condition $\Delta \tau \leq h / \max _{\Omega}\left|j^{\varepsilon}\left(y^{k}, p^{k}\right)\right|$. Finally, in order for the pseudo-time step $\Delta \tau$ to decrease with respect to the iteration $k$, we consider the following pseudo-time step:

$$
\begin{aligned}
\Delta \tau^{k} & =\min \left(1, \max _{\Omega}\left|j^{\varepsilon}\left(y^{k}, p^{k}\right)\right|\right) \frac{h}{\max _{\Omega}\left|j^{\varepsilon}\left(y^{k}, p^{k}\right)\right|} \\
& \leq \Delta \tau^{k-1}, \quad \forall k>0 .
\end{aligned}
$$

Remark 6. The upwind scheme (59) is motivated by the propagation of information through characteristics in the first-order hyperbolic equation (44). Very interestingly with respect to the discussion of the previous section, this scheme may be replaced by usual centered finite differences, provided that an artificial viscosity term is added (see (Osher and Sethian, 1988)), namely, the approximation of $\psi_{\tau}+F|\nabla \psi|=h \Delta \psi$ instead of (44). However, the reason to introduce this term here is different.

4.3. Optimization algorithm. The algorithm to numerically solve Problem $\left(P_{\omega, L}^{\varepsilon}\right)$ may be structured as follows:

1. Meshing once the entire fixed domain $\Omega$. Initialization of the level-set $\psi_{0}$ corresponding to an initial guess $\omega_{0}$ (obtained, for instance, using (34) or (36)).

2. Iteration until convergence, for $k \geq 0$ :

- Computation on $\Omega$ of the state $y^{k}$, the solution of the forward wave system (1) using the scheme 55).

- Computation of the adjoint state $p^{k}$, the solution of the backward wave system (14) using the scheme (57).

- Computation on $\Omega$ of the integrand $j^{\varepsilon}\left(y^{k}, p^{k}\right)$ (see Theorem 1) using the approximation (58).

- Deformation of the shape by solving the transport Hamilton-Jacobi system (45) using the scheme (59). The new domain $\omega_{k+1}$ is characterized by the level-set function $\psi^{k+1}$ being the solution of (45) after a pseudo-time step $\Delta \tau^{k}$ starting from the initial condition $\psi^{k}$ with velocity $-j^{\varepsilon}\left(y^{k}, p^{k}\right)$. The pseudo-time step $\Delta \tau^{k}$ is chosen according to (60). The value $\Delta \tau^{k}$ monitored by the stability condition (60) is usually small enough to ensure a decrease in the cost function.

3. From time to time, for stability reasons, we reinitialize the level-set function $\psi$ by solving (46) using a scheme analogous to 59.

Since, for each iteration, the computation of the state $y^{k}$ and the adjoint $p^{k}$ is much more expensive in CPU time than the resolution of the Hamilton-Jacobi system, we perform several explicit pseudo-time steps of (45) after each resolution of (1) and (14). During these explicit pseudotime steps, we perform regularly some re-initialization of the level set function by solving (46).

Remark 7. One of the main advantages of the level-set method is easy handling of topology changes, i.e., merging and cancellation of holes. Under the strict stability condition (60), the algorithm cannot create holes. This is a consequence of the maximum principle associated with the solution of (45). Therefore, the only possible mechanism is that an initial hole splits into two new holes. This phenomenon will appear in one example developed in Section 5.

The algorithm used to solve Problem $\left(P_{a}\right)$ has a similar structure. The integrand is obtained by approximating (47) or 49).

Finally, we will consider the optimization of the energy with respect to $\omega$ and $a$ simultaneously, i.e.,

$$
\inf _{\omega \in V_{L}, a \in L^{\infty}\left(\Omega, \mathbb{R}^{+}\right)} E^{\varepsilon}(\omega, a, T) .
$$

An iteration of the gradient algorithm may be then written as follows:

$$
\begin{aligned}
\left(\begin{array}{c}
\omega_{k+1} \\
a_{k+1}
\end{array}\right)= & \left(\begin{array}{c}
\omega_{k} \\
a_{k}
\end{array}\right)-\left(\begin{array}{c}
\eta_{1} \boldsymbol{\nu}_{k} \\
\eta_{2}
\end{array}\right) \\
& \cdot\left(\begin{array}{c}
\varepsilon^{-1}\left(\left|\omega_{k}\right|-L|\Omega|\right)+\int_{0}^{T} a_{k} y_{k}^{\prime} p_{k} \mathrm{~d} t \\
\int_{0}^{T} \int_{\omega_{k}} y_{k}^{\prime} p_{k} \mathrm{~d} x \mathrm{~d} t
\end{array}\right)
\end{aligned}
$$

with $\eta=\left(\eta_{1}, \eta_{2}\right)$ small enough.

\section{Numerical experiments}

We now present some numerical simulations. In all the computations, the domain $\Omega$ is the unit square. A uniform mesh is used with $h=1 / 150$ and $\Delta t=h / \sqrt{2}$. We first consider smooth initial conditions, and then some irregular ones in order to stress the necessity of the additional viscosity term in the discretization of the wave system.

\subsection{Regular initial conditions.}

5.1.1. Minimization with respect to $\boldsymbol{\omega}$. We assume that the function $a$ is fixed. We first consider the following regular initial condition function of the first frequency component:

$$
\left(y_{0}(\boldsymbol{x}), y_{1}(\boldsymbol{x})\right)=\left(100 \sin \left(\pi x_{1}\right) \sin \left(\pi x_{2}\right), 0\right),
$$

$\boldsymbol{x}=\left(x_{1}, x_{2}\right) \in \Omega$. We take $L=1 / 10, \varepsilon=10^{-5}, T=1$ and $a(\boldsymbol{x})=10 \mathcal{X}_{\omega}(\boldsymbol{x})$. Before giving numerical results, 
let us apply the relation (36). Let $\alpha=\sqrt{2} \pi$. The conservative solution associated with $\left(y_{0}, y_{1}\right)$ is $y_{\omega, 0}(\boldsymbol{x}, t)=$ $\cos (\alpha t) y_{0}(\boldsymbol{x})$ so that

$$
\left\{\begin{aligned}
\left(p_{\omega, 0}(\boldsymbol{x}, t), p_{\omega, 0}^{\prime}(\boldsymbol{x}, t)\right) & \\
= & \left(\alpha \sin (\alpha T), \alpha^{2} \cos (\alpha T)\right) y_{0}(\boldsymbol{x}), \\
p_{\omega, 0}(\boldsymbol{x}, t)= & \alpha(\sin (\alpha T) \cos (\alpha(t-T)) \\
& +\cos (\alpha T) \sin (\alpha(t-T))) y_{0}(\boldsymbol{x})
\end{aligned}\right.
$$

and

$$
\begin{aligned}
\int_{0}^{T} y^{\prime}(\boldsymbol{x}, t) p & (\boldsymbol{x}, t) \mathrm{d} t \\
& =-\frac{\alpha}{4}(2 \alpha T-\sin (2 \alpha T)) y_{0}(\boldsymbol{x})^{2}<0 .
\end{aligned}
$$

From the relation (36), we have

$$
\begin{aligned}
& E(\omega, a, T)-E(\omega, 0, T) \\
& =-\frac{a \alpha}{4}(2 \alpha T-\sin (2 \alpha T)) \int_{\omega}\left(y_{0}(\boldsymbol{x})\right)^{2} \mathrm{~d} x+o(a), \\
& \forall T \geq 0 .
\end{aligned}
$$

For $a$ small enough and for all $T>0$, the dissipation is then optimal for $\omega$ which maximizes the integral $\int_{\omega}\left(y_{0}(\boldsymbol{x})\right)^{2} \mathrm{~d} x$, i.e., centered on the unit square $\Omega$. The simulations confirm this prediction. Figure 2 illustrates the evolution of the zero-level set $\left\{\boldsymbol{x} \in \Omega, \psi_{k}(\boldsymbol{x})=0\right\}=$ $\partial \omega_{k}$ with respect to the iteration $k$ (equivalently, with respect to the pseudo-time parameter $\tau>0$ ). Let us denote by $\omega_{(c)}$ the disc with radius $\sqrt{L / \pi}$ and center $(c, c)$. The sequence of domains, $\omega_{k}$, is initialized by $\omega_{0}=\omega_{(0.35)}$ so that $\left|\omega_{0}\right|=L$ (the boundary $\partial \omega_{0}$ is marked with the dash-dot line in Fig 2). Figure 3 depicts the cost function and $\left|\omega_{k}\right|$ with respect to $k$. As expected, the limit of the sequence $\left(\omega_{k}\right)_{k>0}$ is near a disc centered at $(1 / 2,1 / 2)$. We check that we obtain exactly a disc when $\Omega$ is itself a disc. We also observe that the value of $\varepsilon=10^{-5}$ is small enough to maintain $\left|\omega_{k}\right|$ close to $L$. This result is invariant with respect to $T$ and also with respect to the initialization $\omega_{0}$.

Figures 4 and 5 display the evolution of $\partial \omega_{k}$ when $\omega_{0}$ is composed of 4 and 9 disjoints discs, respectively. Figures 6 and 7 display the evolution of the corresponding area and cost. The limit cost functions obtained with these three initializations are very similar. Let us now consider the initial condition $y_{0}(\boldsymbol{x})=100 \sin \left(2 \pi x_{1}\right) \sin \left(\pi x_{2}\right)$ for which $y_{\omega, 0}(\boldsymbol{x}, t)=100 \cos (\sqrt{5} \pi t) \sin \left(2 \pi x_{1}\right) \sin \left(\pi x_{2}\right)$. The relation 65 still holds for $\alpha=\sqrt{5} \pi$ showing that, for $a$ small enough, the optimal position of $\omega$ is related to the points $(1 / 4,1 / 2)$ and $(3 / 4,1 / 2)$ where the function $y_{0}^{2}$ admits two maxima. Once again, the numerical simulations are in good agreement with this prediction. Figures 8 and 9 display the evolution of the sequence $\left(\omega_{k}\right)_{k>0}$ when $\omega_{0}$ is composed of one and one hundred disjoints parts, respectively. For these examples, the invariance with respect to $\omega_{0}$ illustrates (for small $a$ ) the uniqueness of the minimum.

On the other hand, with more general initial conditions (for instance, without symmetries in space), the limit of the sequence $\left(\omega_{k}\right)_{k>0}$ may depend on both $\omega_{0}$ and $T$, highlighting the presence of local minima. In this case, we observe that an initialization $\omega_{0}$ composed of several components provides a lower value of the cost (i.e., better local minima). Figure 10 displays the limit of $\partial \omega_{k}$ for $T=1$ and $T=2$ and four configurations of $\omega_{0}$ when $y_{0}(\boldsymbol{x})=300 x_{1} x_{2}\left(x_{1}-1\right)\left(x_{2}-1\right) \cos \left(5 \pi x_{1}\left(x_{2}-\right.\right.$ 1)) $\sin \left(2 \pi x_{1} x_{2}\right)$ and $y_{1}=0$. Table 10 gives the energy $E(\omega, a, T)$ associated with these limits. Remark that the existence of several local minima does not necessarily imply that Problem $\left(P_{\omega}\right)$ is ill posed: since a finite number of frequency components is present in $y_{0}$ and since $a$ is small, we may conjecture that the optimal design is composed of a finite number of disjoint components. For these data, Fig 11 depicts the topological derivative $\boldsymbol{x} \rightarrow \int_{0}^{T} y_{\emptyset, 0}^{\prime}(\boldsymbol{x}, t) p_{\emptyset, 0}(\boldsymbol{x}, t) \mathrm{d} t$ in $\Omega$ (see Eqn. (36)); the corresponding characteristic function (at the bottom) of size $L$ illustrates how this derivative provides an efficient initialization (we refer to (Allaire et al., 2005; Fulmanski et al., 2008) for a strong coupling between the level set and the topological derivative).

Table 1. $E(\omega, a=10 ., T)$ for different initial predictions $\omega_{0}$ Here $\sharp \omega_{0}$ is the number of disjoint parts of $\omega_{0}$ (associated with Fig. 10].

\begin{tabular}{c|cccc}
\hline$T$ & $\sharp \omega_{0}=1$ & $\sharp \omega_{0}=9$ & $\sharp \omega_{0}=25$ & $\sharp \omega_{0}=49$ \\
\hline 1 & 502.64 & 261.88 & 256.86 & 249.10 \\
2 & 322.88 & 117.99 & 96.53 & 88.17 \\
\hline
\end{tabular}

\subsubsection{Minimization with respect to the damping} function $\boldsymbol{a}$. We consider once again the initial condition $y_{0}(\boldsymbol{x})=100 \sin \left(\pi x_{1}\right) \sin \left(\pi x_{2}\right), y_{1}=0$, fix $\omega$ and optimize with respect to $a$. Figure 12 represents the energy $E\left(\omega_{(1 / 2)}, a, 1\right)$ with respect to the constant $a$ on $\omega=\omega_{(1 / 2)}$. The minimum of the energy with respect to $a$ is obtained for $a \approx 15.33$ and $E\left(\omega_{(1 / 2)}, 15.33,1\right) \approx$ 439.59. The value 15.33 is obtained using the descent direction (47). Figure 12 illustrates the over-damping phenomenon: since $y_{1}=0, E(\omega, a, T=1)$ converges towards $E(\omega, a, 0)=E(\emptyset, 0,0)=E(\omega, 0, T)$ as $a$ tends to infinity. Numerically, we obtain $E(\omega, 0, T)-$ $E(\omega, a, T) \approx O\left(a^{-0.78}\right)$

Obviously, the value 439.59 is improved if we assume that the function $a$ may depend on $x$ in $\omega$. Figure 13 describes the graph of $a_{k}\left(x_{1}, 1 / 2\right), x_{1} \in[0,1]$ for different iterations $k$ of the descent algorithm using the 

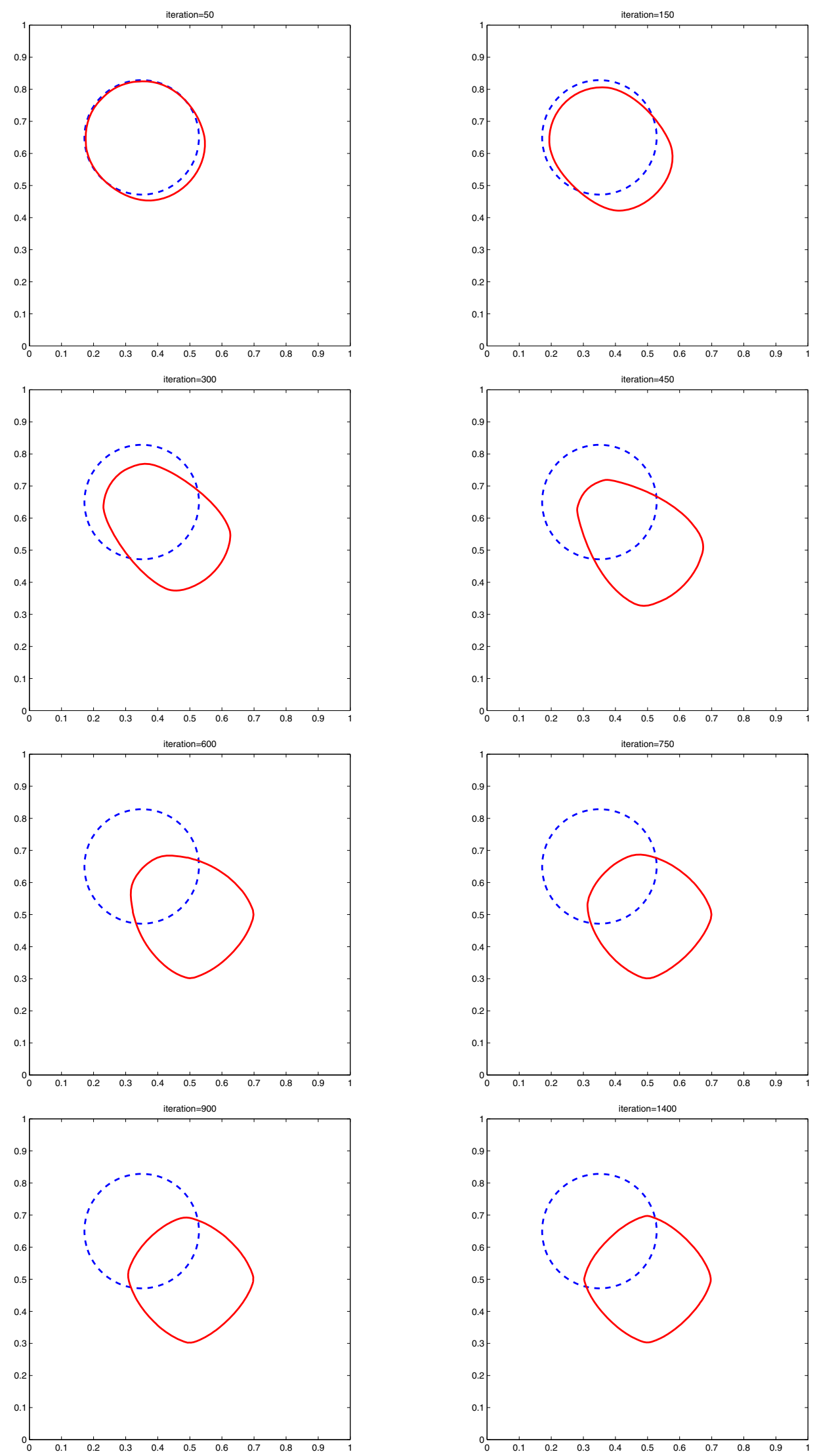

Fig. 2. $\left(y_{0}, y_{1}\right)=\left(100 \sin \left(\pi x_{1}\right) \sin \left(\pi x_{2}\right), 0\right), T=1, a=10$. Evolution of the zero-level set $\left\{\boldsymbol{x} \in \Omega, \psi_{k}(\boldsymbol{x})=0\right\}$ with respect to $k$. 

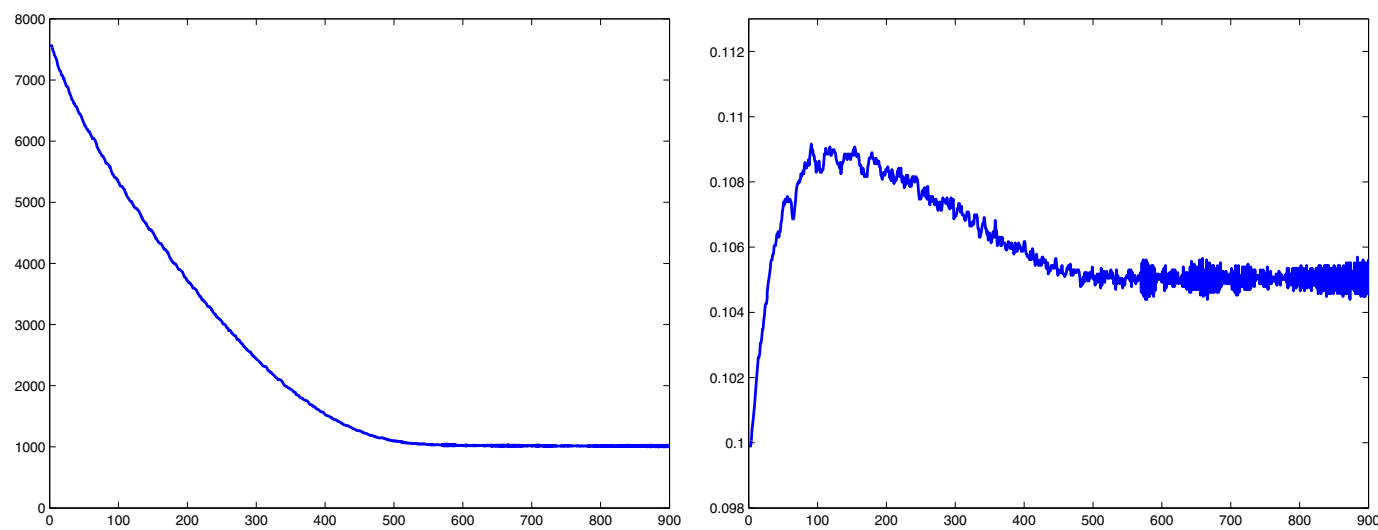

Fig. 3. $\left(y_{0}, y_{1}\right)=\left(100 \sin \left(\pi x_{1}\right) \sin \left(\pi x_{2}\right), 0\right), T=1$. Evolution of $E\left(\omega_{k}, a, T\right)$ (left) and $\left|\omega_{k}\right|$ (right) with respect to $k$ (associated with Fig. 2].

direction 49]. We observe, in agreement with the theoretical analysis in (Freitas, 1999; López-Gómez, 1997), that the functions $a_{k}(\boldsymbol{x})$ take negative values in $\omega$. We also observe that the norm $\left\|a_{k}\right\|_{L^{\infty}(\omega)}$ is not bounded with respect to $k$. Precisely, on the boundary of $\omega$, the function $a_{k}$ tends to infinity with $k$. This simulation suggests that the derivative of the damping potential is as important as its sign for the dissipation. This is in agreement with (Castro and Cox, 2001), where it is shown that the unbounded function $a(x)=1 / x$ in $(0,1)$ extinguishes in finite time the solution of the 1-d damped wave equation. We remark, however, that the average $\int_{\omega} a_{k}(\boldsymbol{x}) \mathrm{d} x /|\omega|$ remains bounded and converges as $k$ tends to infinity to the value 15.33 (optimal for $a$ constant in $\omega$ ). These observations illustrate the influence of the over-damping phenomenon and the richness of Problem $\left(P_{a}\right)$.

The optimal value of $a$ also depends on the domain $\omega$. For instance, for $\omega=\omega_{(0.35)}$, the optimal damping constant is $a \approx 24.89$ leading to $E\left(\omega_{(0.35)}, 24.89,1\right) \approx$ 4916.93

Very interestingly, if we now come back to Problem $\left(P_{\omega}\right)$ with a value larger than $a=10$ (used in Section 5.1.1), e.g., $a=25$ and $\omega_{0}=\omega_{(0.35)}$, we do not obtain a centered disc anymore. The limit in $k$ of the zerolevel set $\left\{\boldsymbol{x} \in \Omega, \psi_{k}(\boldsymbol{x})=0\right\}$ is depicted in Fig. 14 (top left) leading to a cost $E\left(\omega_{(0.35)}, a=25, T\right) \approx 101.08$ (in comparison with $E\left(\omega_{(1 / 2)}, 15.33, T\right) \approx 439.59<$ $\left.E\left(\omega_{(1 / 2)}, a=25, T\right)\right)$. At the limit, the domain $\omega$ is divided into three parts! Note that this point is not in contradiction with the relation (65) because the value $a=25$ is not "small" enough. We remark that this value is in the increasing part of the function $a \rightarrow E(\omega, a, T)$ (see Fig. 12) whereas the value $a=10$ used in Section 5.1.1 is in the decreasing part. Moreover, for this value, the invariance with respect to the initialization $\omega_{0}$ is lost. Figure 14 depicts the limit in $k$ of the zero-level set $\left\{\boldsymbol{x} \in \Omega, \psi_{k}(\boldsymbol{x})=0\right\}$ for three other initial predictions $\omega_{0}$. Even for a symmetric $\omega_{0}$ (Fig. 14, bottom right), the limit is not a centered disc but is associated with a limit cost function $E\left(\omega_{k}, a, T\right) \approx 50.35$ significantly smaller than $E\left(\omega_{(1 / 2)}, a=25, T\right) \approx 1278.48$. This highlights the existence of several local minima. Actually, for this value, Problem $\left(P_{\omega}\right)$ is ill posed (Münch et al., 2006), and the optimal domain is composed of an arbitrary number of disjoint components distributed in $\omega$ : this phenomenon may be numerically detected by taking a sequence (in $p$ ) of initial guesses $\left(\omega_{0, p}\right)_{(p)}$ with an increasing number of disjoint components $\sharp \omega_{0, p}$ : if the corresponding sequence $\sharp \omega_{k, p}$, associated with the limit sequence of domains $\left(\omega_{k, p}\right)_{p}$, increases, then the ill-posedness is likely to hold.

5.1.3. Minimization with respect to both $\omega$ and $a(x)$. Using the algorithm 62, we now minimize the cost function with respect to both $\omega$ and $a$. According to our previous observations, these two variables are strongly coupled. We simply consider the case of $a$ constant in $\omega$. Figure 15 associated with $\left(y_{0}, y_{1}\right)=$ $\left(100 \sin \left(\pi x_{1}\right) \sin \left(\pi x_{2}\right), 0\right.$.), depicts the limit of the zerolevel set sequence $\left\{\boldsymbol{x} \in \Omega, \psi_{k}(\boldsymbol{x})=0\right\}$ obtained for different initializations $\omega_{0}$ and $a_{0}=10$, whereas Fig. 16 depicts the evolution of $a_{k}$ with respect to $k$. The results are summarized in Table 2] Except for $\omega_{0}=\omega_{(0.35)}$ and $a_{0}=$ 10 (see Fig. 16 top left) leading to $E\left(\omega_{k}, a, T\right) \approx 140.12$, the minimization with respect to both $\omega$ and $a$ leads to an impressive reduction of the cost function. Once again, the result depends on the initial values $\left(\omega_{0}, a_{0}\right)$ and is improved when $\omega_{0}$ is composed of several disjoints parts. Finally, a better reduction is observed when the function $a$ varies in $\omega$. Similar results are obtained with the viscous schemes (55) and (57).

5.1.4. Interplay between the values of $a$ and $|\omega|$ and the topological derivative. The previous numeri- 

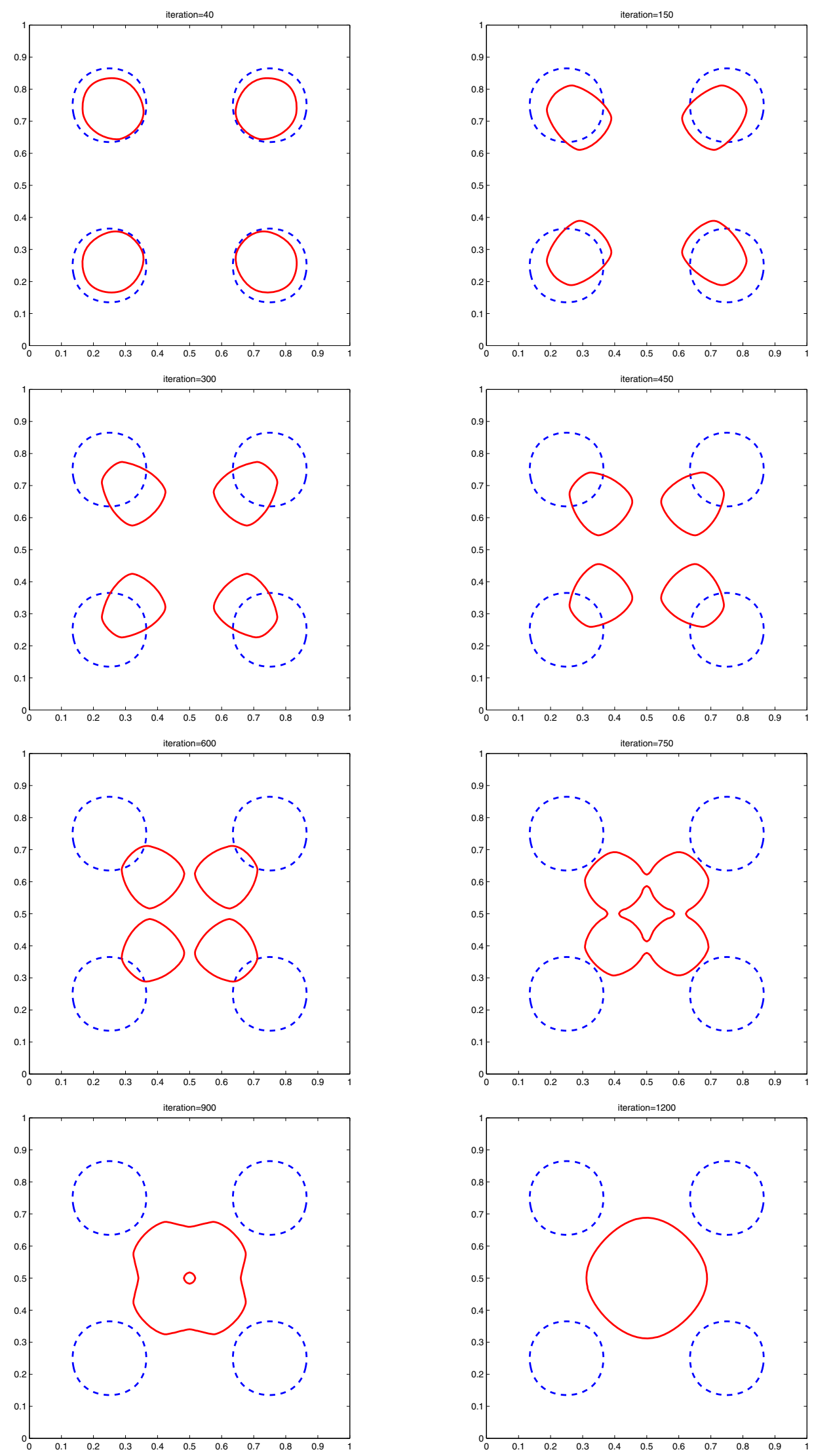

Fig. 4. $\left(y_{0}, y_{1}\right)=\left(100 \sin \left(\pi x_{1}\right) \sin \left(\pi x_{2}\right), 0\right), T=1, a=10$. Evolution of the zero-level set $\left\{\boldsymbol{x} \in \Omega, \psi_{k}(\boldsymbol{x})=0\right\}$ with respect to $k$. 

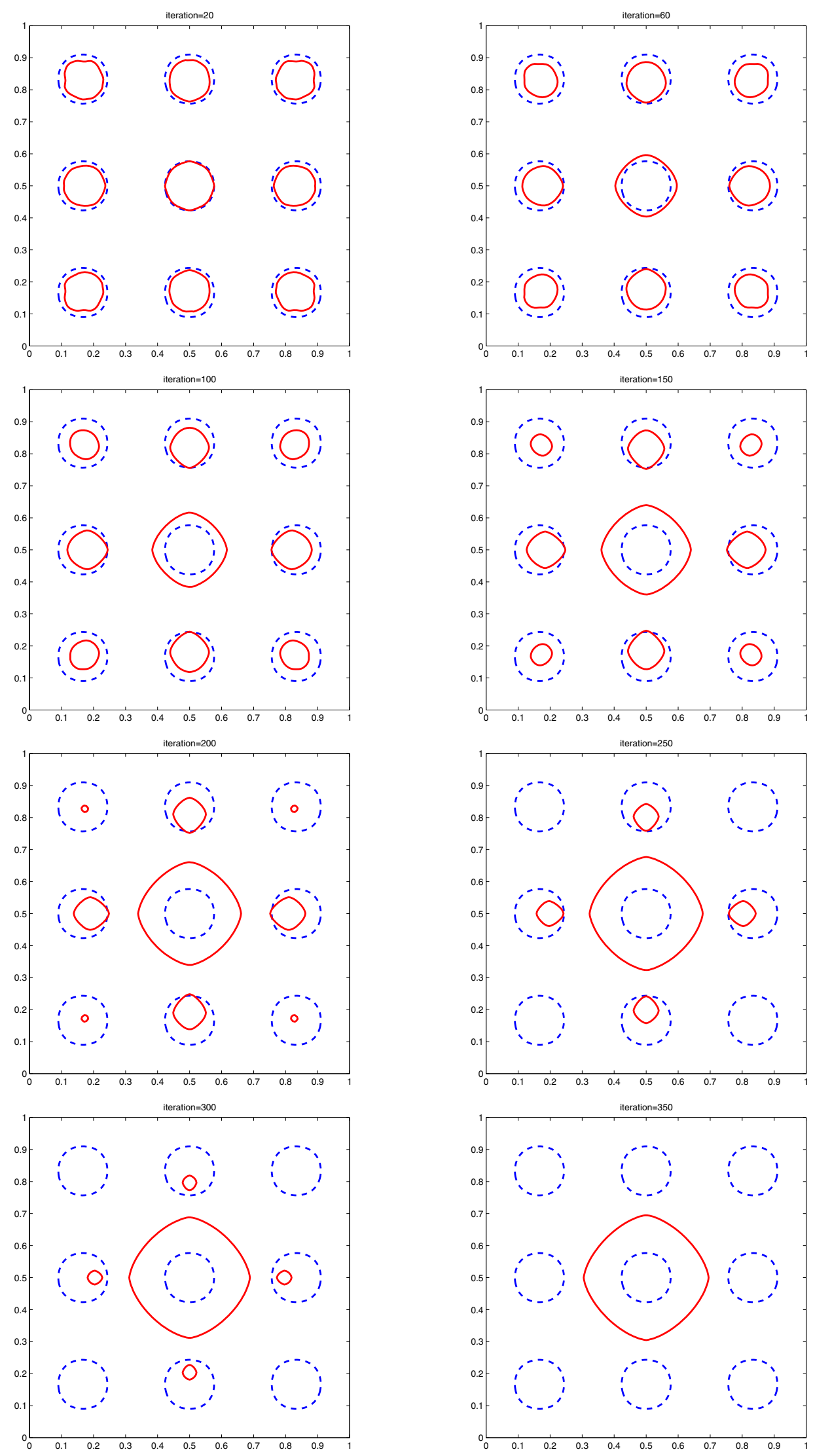

Fig. 5. $\left(y_{0}, y_{1}\right)=\left(100 \sin \left(\pi x_{1}\right) \sin \left(\pi x_{2}\right), 0\right), T=1, a=10$. Evolution of the zero-level set $\left\{\boldsymbol{x} \in \Omega, \psi_{k}(\boldsymbol{x})=0\right\}$ with respect to $k$. 

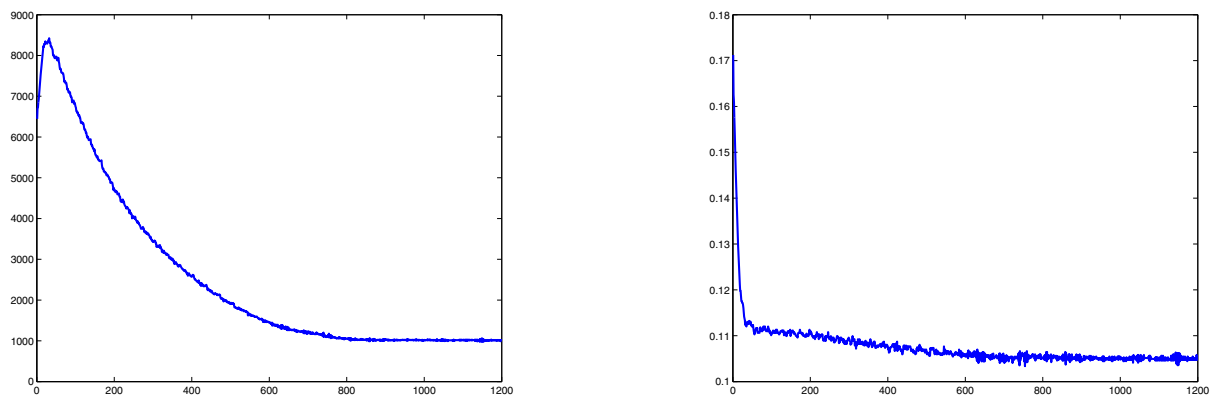

Fig. 6. $\left(y_{0}, y_{1}\right)=\left(100 \sin \left(\pi x_{1}\right) \sin \left(\pi x_{2}\right), 0\right), T=1, a=10$. Evolution of $E\left(\omega_{k}, a, T\right)$ (left) and $\left|\omega_{k}\right|$ (right) with respect to $k$ (associated with Fig. 44.
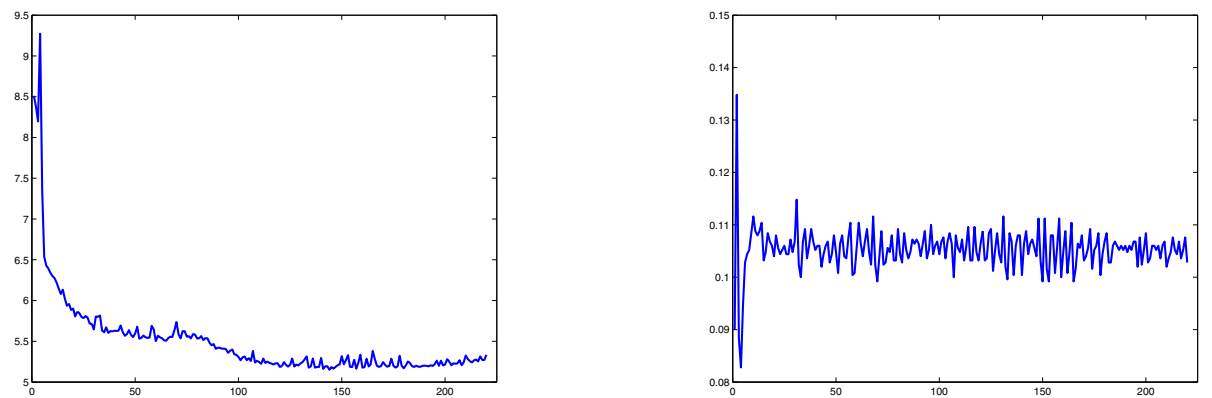

Fig. 7. $\left(y_{0}, y_{1}\right)=\left(100 \sin \left(\pi x_{1}\right) \sin \left(\pi x_{2}\right), 0\right), T=1, a=10$. Evolution of $E\left(\omega_{k}, a, T\right)$ (left) and $\left|\omega_{k}\right|$ (right) with respect to $k$ (associated with Fig. 5 .

Table 2. $E(\omega, a, T=1)$ for different initial predictions $\omega_{0}$. Here $\sharp \omega_{0}$ is the number of disjoint parts of $\omega_{0}$ (associated with Figs. 15 and 16 .

\begin{tabular}{c|cccc}
\hline$\sharp \omega_{0}$ & 1 & 4 & 16 & 49 \\
\hline$E\left(\omega_{2000}, a_{2000}, 1\right)$ & 140.12 & 12.54 & 17.79 & 15.83 \\
\hline$a_{2000}$ & 19.51 & 29.09 & 35.12 & 29.38 \\
\hline
\end{tabular}

cal simulation suggest that, for fixed $|\omega|$, Problem $\left(P_{\omega}\right)$ is not well posed as soon as $a$ exceeds a critical value, $a_{c}$ say. This critical value depends, among other things, on the size $|\omega|$. As already explained at the beginning of Section 5.1.1, these points (related to the over-damping phenomenon) may be observed from the topological derivative. Let us consider the initial data (63), $L=|\omega|=1 / 10$ and $T=1$. According to Section 5.1.1 and (36), if $a$ is small enough, then the optimal position is the centered disc $D\left(\boldsymbol{x}_{\mathbf{0}}, \rho\right) \subset \Omega$ for any $\rho$ with $\boldsymbol{x}_{\mathbf{0}}=(1 / 2,1 / 2)$. Similarly, from the relation (34), if $|\omega|$ (equivalently $\rho$ ) is small enough, the disc $D\left(\boldsymbol{x}_{\mathbf{0}}, \rho\right)$ is optimal for any value of $a$, so that $a_{c}$ is a decreasing function of $|\omega|$. This bifurcation of the shape with respect to $a$ may be detected from the computation of the topological derivative. Figure 17 displays the function $\boldsymbol{x} \rightarrow \int_{0}^{T} y_{\omega, a}^{\prime}(\boldsymbol{x}, t) p_{\omega, a}(\boldsymbol{x}, t) \mathrm{d} t$ for $\omega=D\left(x_{0}, L / 4\right)$ and two values of $a=10$ (left), $a=25$ (right). For $a=10$, the derivative enforces the disc $\omega$ to increase smoothly (as expected), while for $a=25$, the centered disc degenerates into a ring. For $a$ large enough, $\omega$ is composed of an arbitrarily large number of disjoints components. Such a structure may be obtained from a relaxation procedure (we refer to (Münch et al., 2006)).

5.2. Irregular initial conditions. So far, we have considered a regular initial condition $\left(y_{0}, y_{1}\right)$ for which (at the numerical level) the use of viscosity terms is unnecessary. In order to highlight the importance of the high frequency component on the mechanism of dissipation, let us consider the most singular situation with a discontinuous initial condition $y_{0}$. We give only one example and refer to (Hébrard and Henrot, 2003) in 1-D. On the unit square $\Omega=(0,1)^{2}$, we define

$$
y_{0}(\boldsymbol{x})= \begin{cases}40 & \left(x_{1}, x_{2}\right) \in(1 / 3,2 / 3)^{2} \\ 0 & \text { elsewhere }\end{cases}
$$

$y_{1}(\boldsymbol{x})=0$ and $a(\boldsymbol{x})=10 \mathcal{X}_{\omega}(\boldsymbol{x})$ and then optimize with respect to $\omega$. Figure 18 displays the limit in $k$ of the boundary of $\omega_{k}$ obtained respectively with the usual schemes without viscosity terms and with the modified viscous schemes (55)-57). The limit is different and 

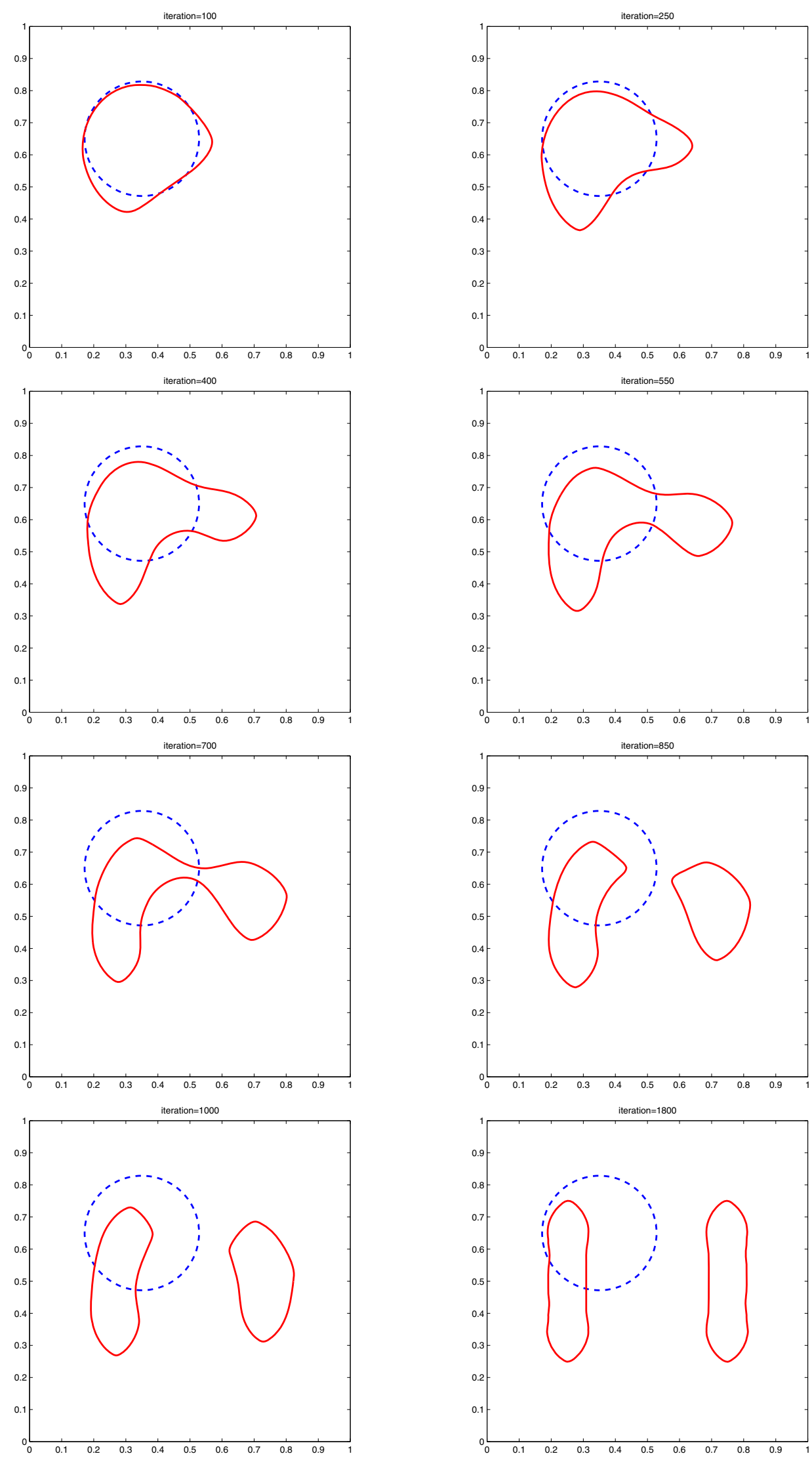

Fig. 8. $\left(y_{0}, y_{1}\right)=\left(100 \sin \left(2 \pi x_{1}\right) \sin \left(\pi x_{2}\right), 0\right), T=1, a=10$. Evolution of the zero-level set $\left\{\boldsymbol{x} \in \Omega, \psi_{k}(\boldsymbol{x})=0\right\}$ with respect to $k$. 

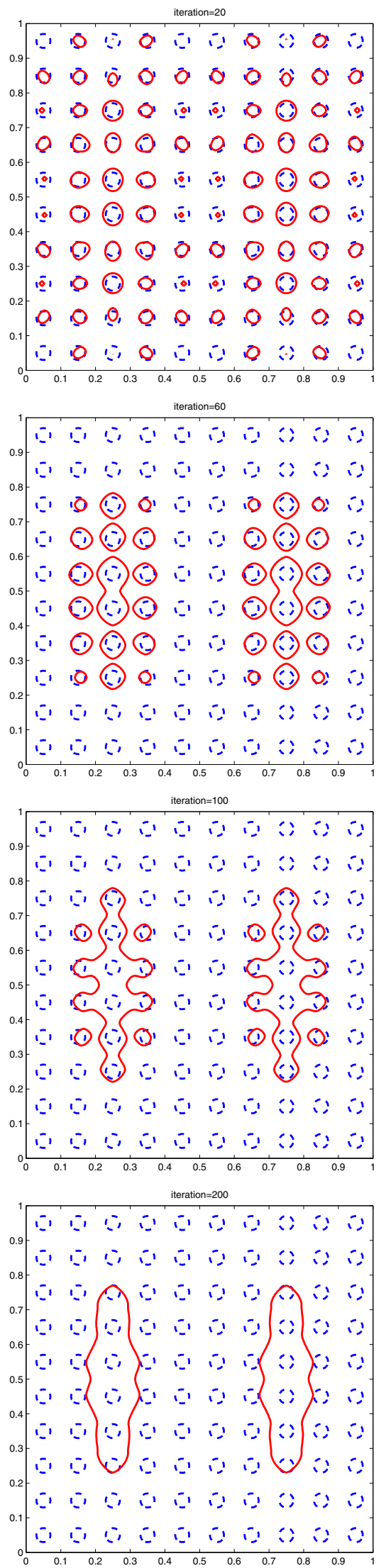
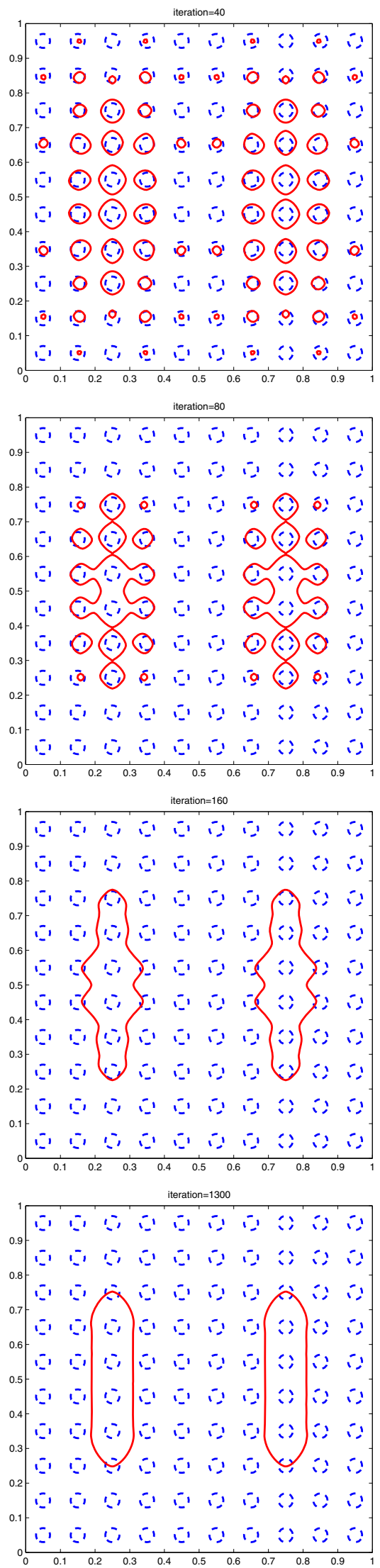

Fig. 9. $\left(y_{0}, y_{1}\right)=\left(100 \sin \left(2 \pi x_{1}\right) \sin \left(\pi x_{2}\right), 0\right), T=1, a=10$. Evolution of the zero-level set $\left\{\boldsymbol{x} \in \Omega, \psi_{k}(\boldsymbol{x})=0\right\}$ with respect to $k$. 


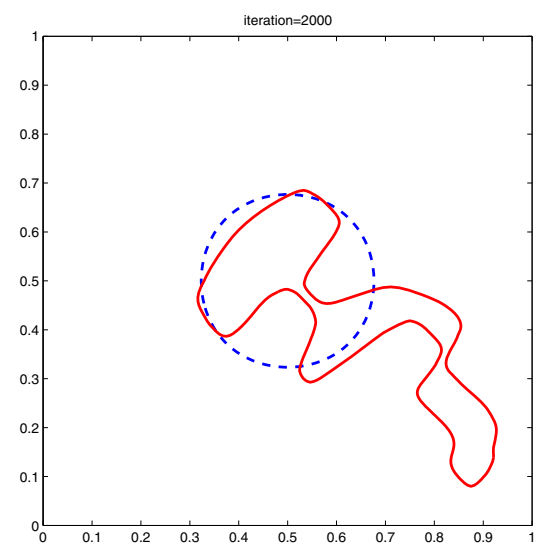

iteration $=2000$

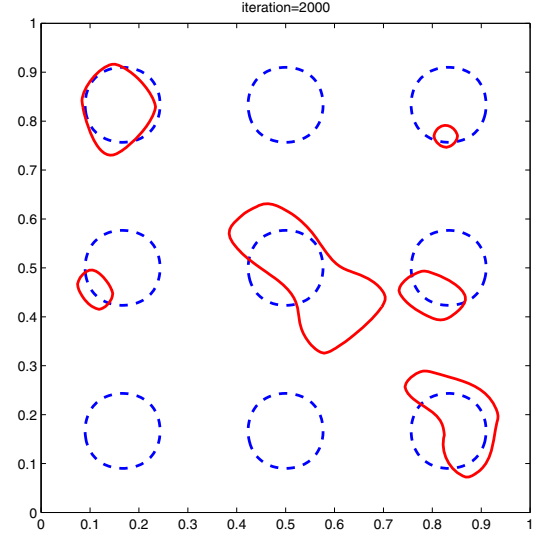

iteration $=1500$
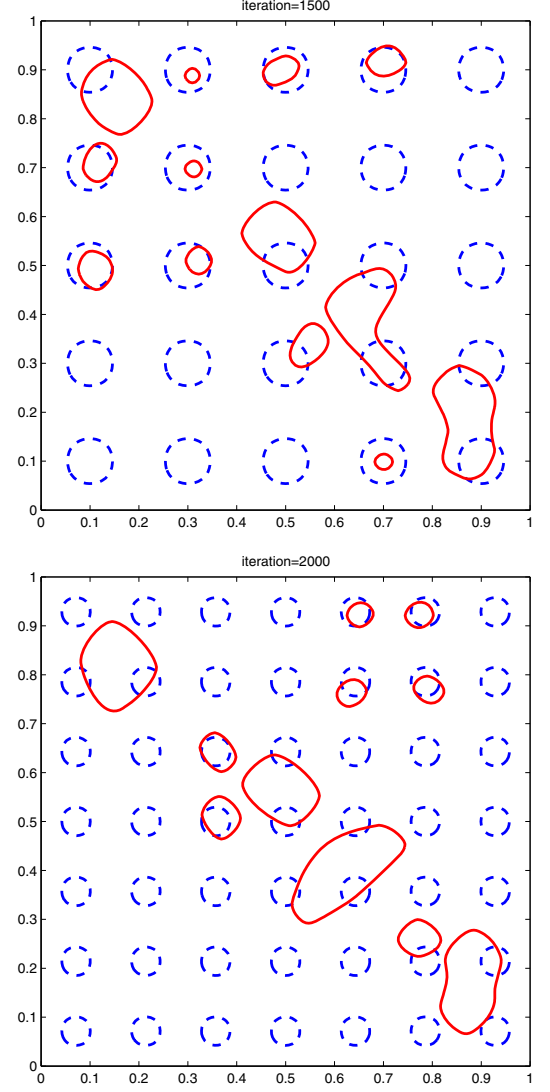

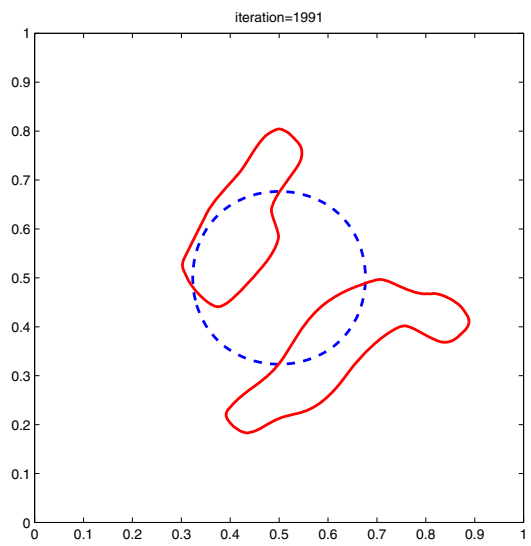

iteration $=2000$

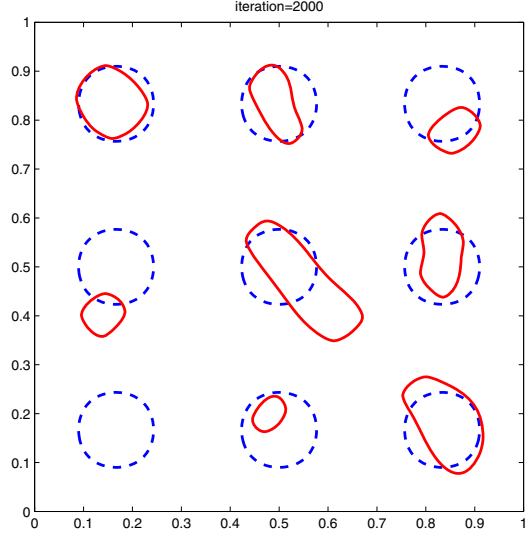

iteration $=1500$
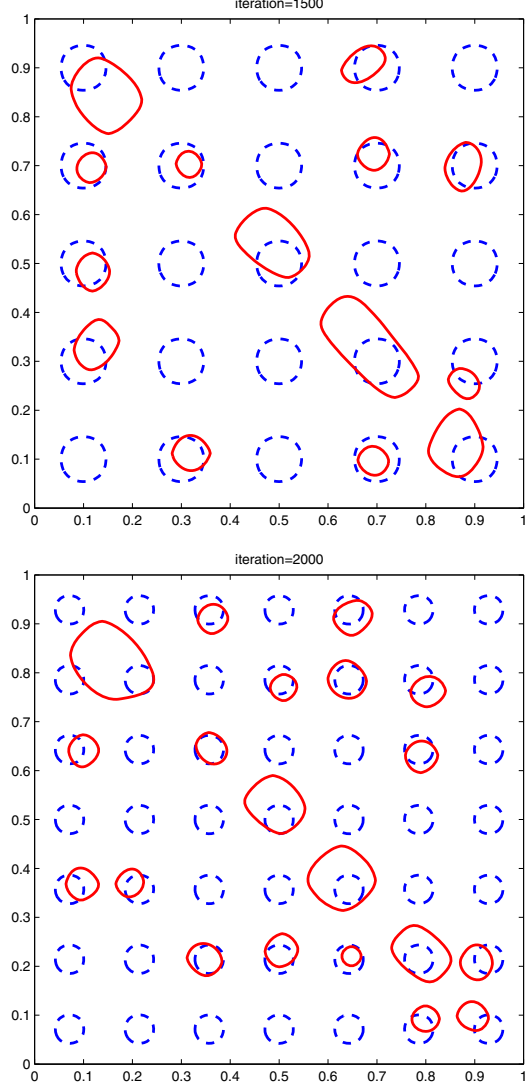

Fig. 10. $\left(y_{0}, y_{1}\right)=\left(300 x_{1} x_{2}\left(x_{1}-1\right)\left(x_{2}-1\right) \cos \left(5 \pi x_{1}\left(x_{2}-1\right)\right) \sin \left(2 \pi x_{1} x_{2}\right), 0\right), a=10$. The "limit" of the sequence the zero-level set $\left\{\boldsymbol{x} \in \Omega, \psi_{k}(\boldsymbol{x})=0\right\}$ for different initial predictions of $\omega_{0}, T=1$ (left) and $T=2$ (right). 

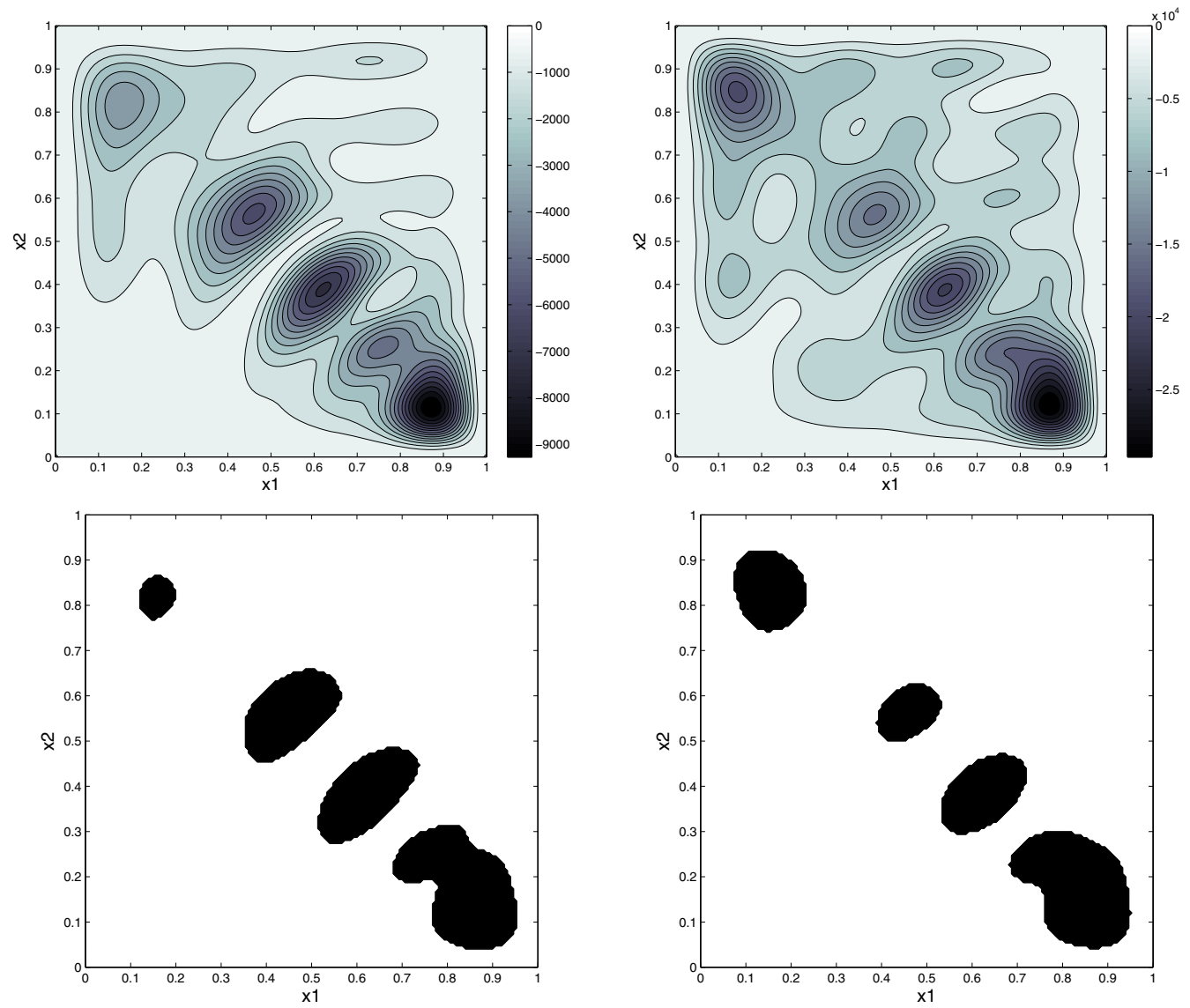

Fig. 11. Case of $\left(y_{0}, y_{1}\right)=\left(300 x_{1} x_{2}\left(x_{1}-1\right)\left(x_{2}-1\right) \cos \left(5 \pi x_{1}\left(x_{2}-1\right)\right) \sin \left(2 \pi x_{1} x_{2}\right), 0\right)$. Topological derivative $\boldsymbol{x} \rightarrow$ $\int_{0}^{T} y_{\emptyset, 0}^{\prime}(\boldsymbol{x}, t) p_{\emptyset, 0}(\boldsymbol{x}, t) \mathrm{d} t$ in $\Omega$ (top) and the corresponding initialization $\omega_{0}$ of size $L$ (bottom), $T=1$ (left) and $T=2$ (right).

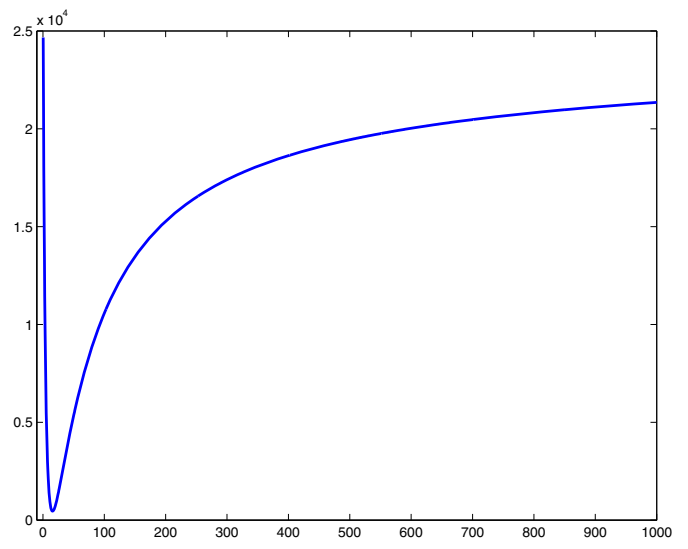

Fig. 12. Illustration of the over-damping phenomenon when $a$ is large: $E(\omega, a, T=1)$ with respect to $a$ (constant on $\omega), \omega$ is the disc with radius $\sqrt{0.1 / \pi}$ centered at $(1 / 2,1 / 2), E(\omega, 0, T)-E(\omega, a, T) \approx O\left(a^{-0.78}\right)$, $a \gg 1$.

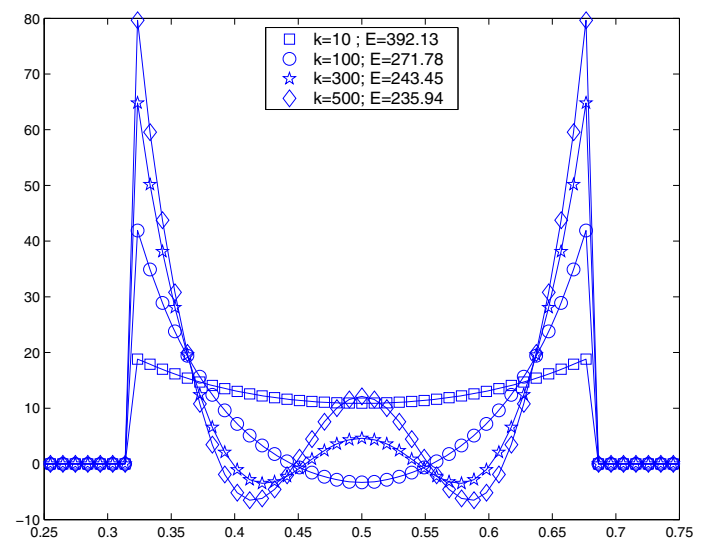

Fig. 13. Optimization of $a(\boldsymbol{x})$ when $\omega$ is the disc of radius $\sqrt{L / \pi}$ centered at $(1 / 2,1 / 2)$. Graph of $a_{k}\left(x_{1}, x_{2}=\right.$ $1 / 2)$ along the axis $\left(O x_{1}\right)$ for different iterations. 

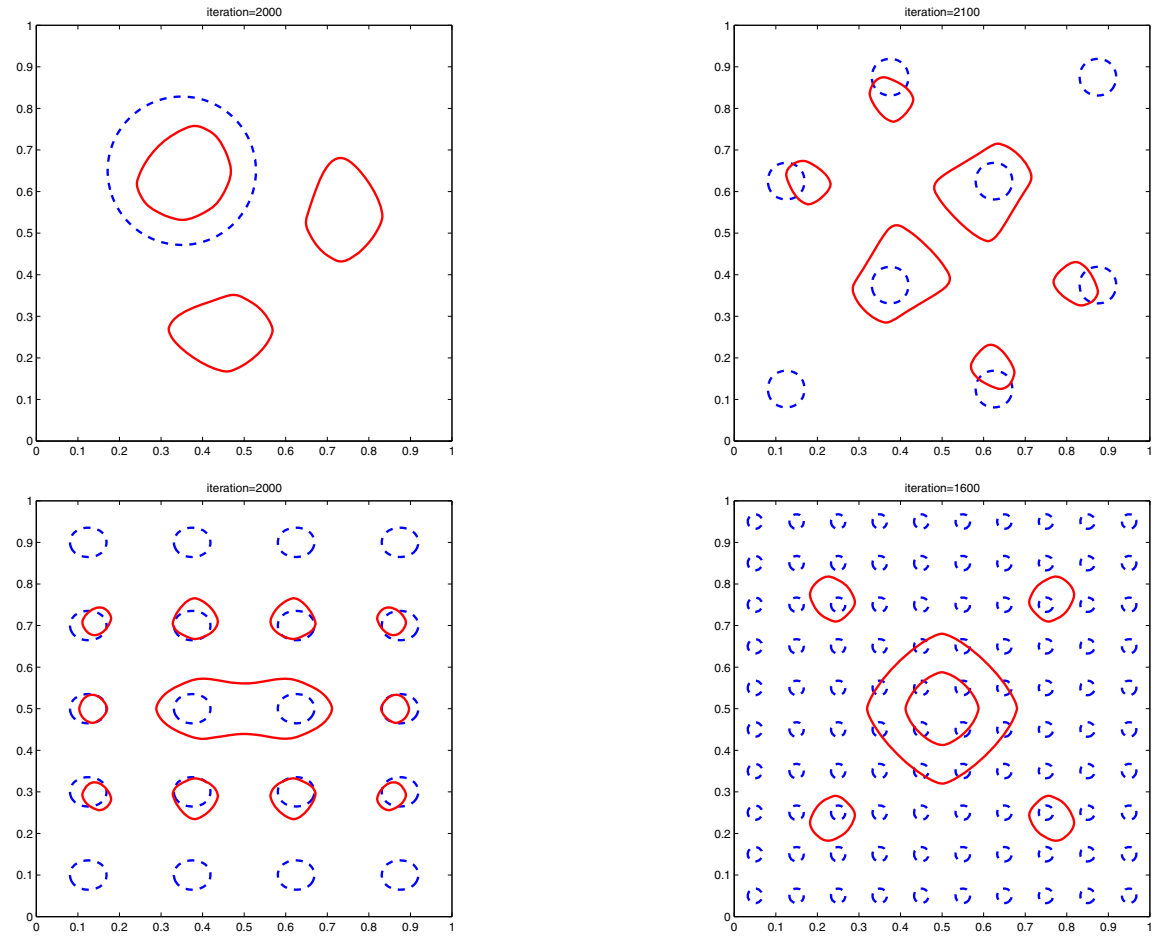

Fig. 14. Case of $\left(y_{0}, y_{1}\right)=\left(100 \sin \left(\pi x_{1}\right) \sin \left(\pi x_{2}\right), 0\right), T=1, a=25$. The "limit" in $k$ of the zero-level set sequence $\{\boldsymbol{x} \in$ $\left.\Omega, \psi_{k}(\boldsymbol{x})=0\right\}$ for different initial predictions $\omega_{0}$. Top left: $E(\omega, a, T)=101.08$, top right: $E(\omega, a, T)=93.47$, bottom left: $E(\omega, a, T)=34.82$, bottom right: $E(\omega, a, T)=50.35$.
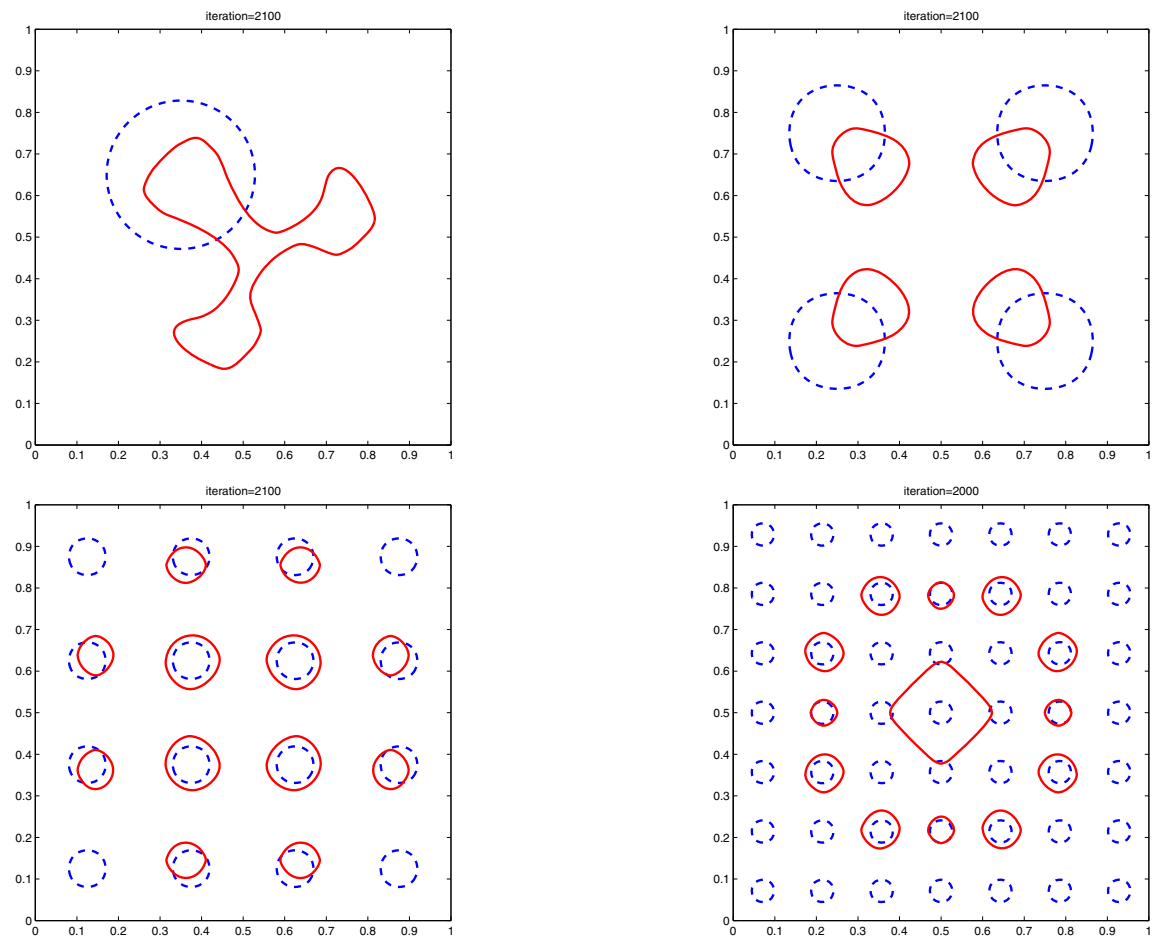

Fig. 15. Case of $\left(y_{0}, y_{1}\right)=\left(100 \sin \left(\pi x_{1}\right) \sin \left(\pi x_{2}\right), 0\right), T=1$. Optimization with respect to $a$ and $\omega-$ The "limit" of the sequence of the zero-level sets $\left\{\boldsymbol{x} \in \Omega, \psi_{k}(\boldsymbol{x})=0\right\}$ for four initial predictions $\omega_{0}$. 

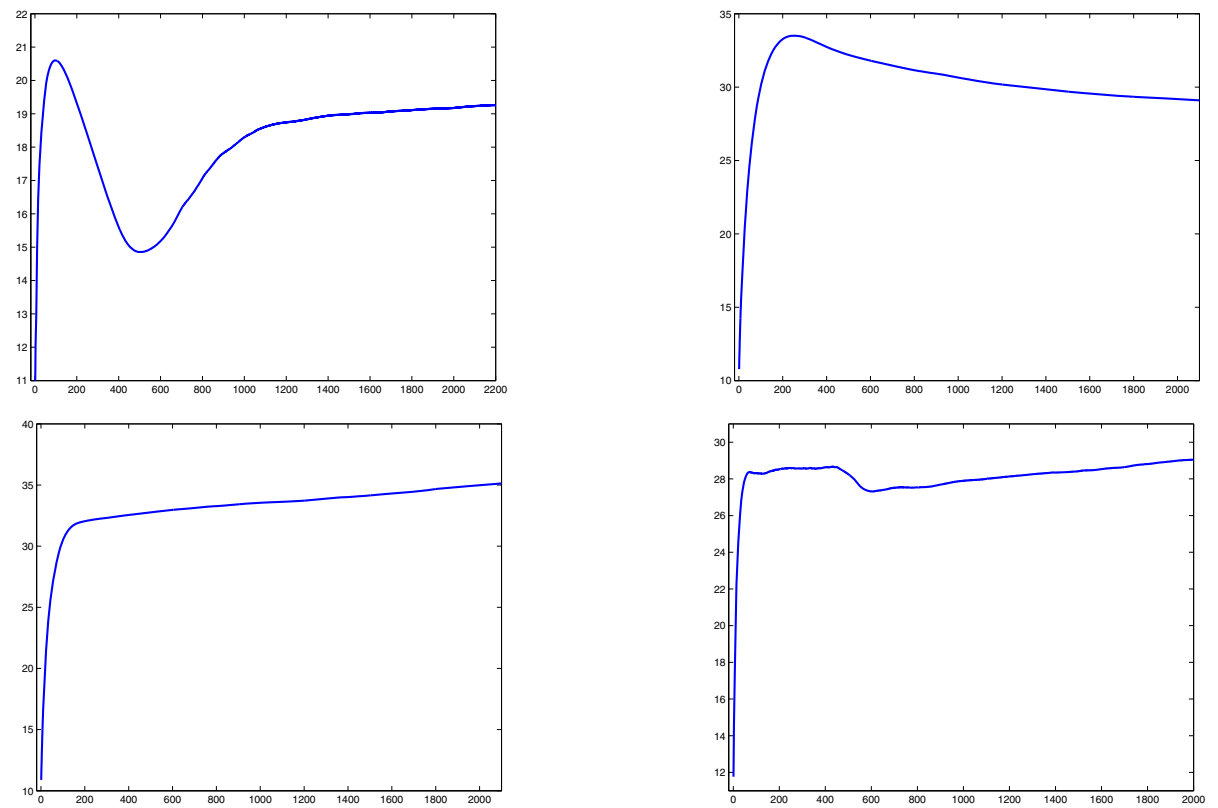

Fig. 16. Case of $\left(y_{0}, y_{1}\right)=\left(100 \sin \left(\pi x_{1}\right) \sin \left(\pi x_{2}\right), 0\right), T=1$. Optimization with respect to $a$ and $\omega$-Evolution of $a_{k}$ with respect to $k$ associated with the initial prediction $\omega_{0}$ of Fig. 15
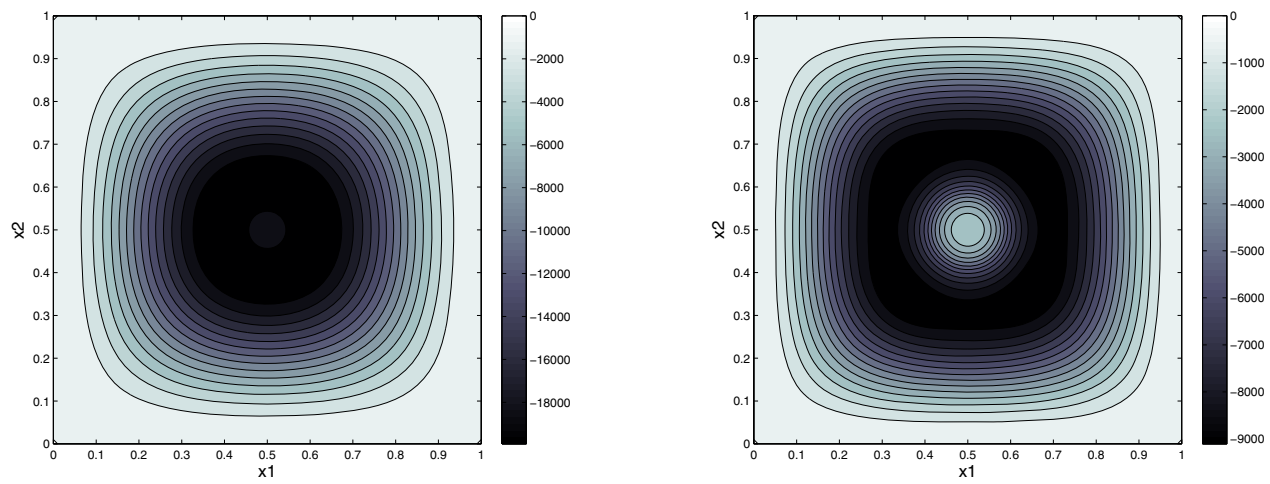

Fig. 17. $\left(y_{0}, y_{1}\right)=\left(100 \sin \left(\pi x_{1}\right) \sin \left(\pi x_{2}\right), 0\right), T=1$.
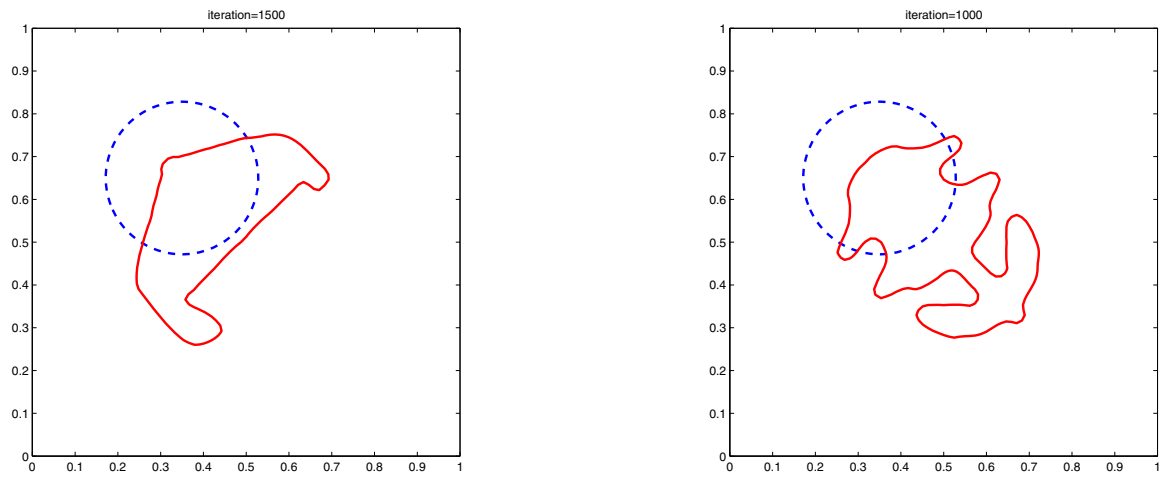

Fig. 18. Singular case: $T=1, a=10$, the "limit" in $k$ of the zero-level set sequence $\left\{\boldsymbol{x} \in \Omega, \psi_{k}(\boldsymbol{x})=0\right\}$ without viscosity terms $E(\omega, a, T) \approx 2768.70$ (left) and with viscosity terms $E(\omega, a, T)=1487.23$ (right). 

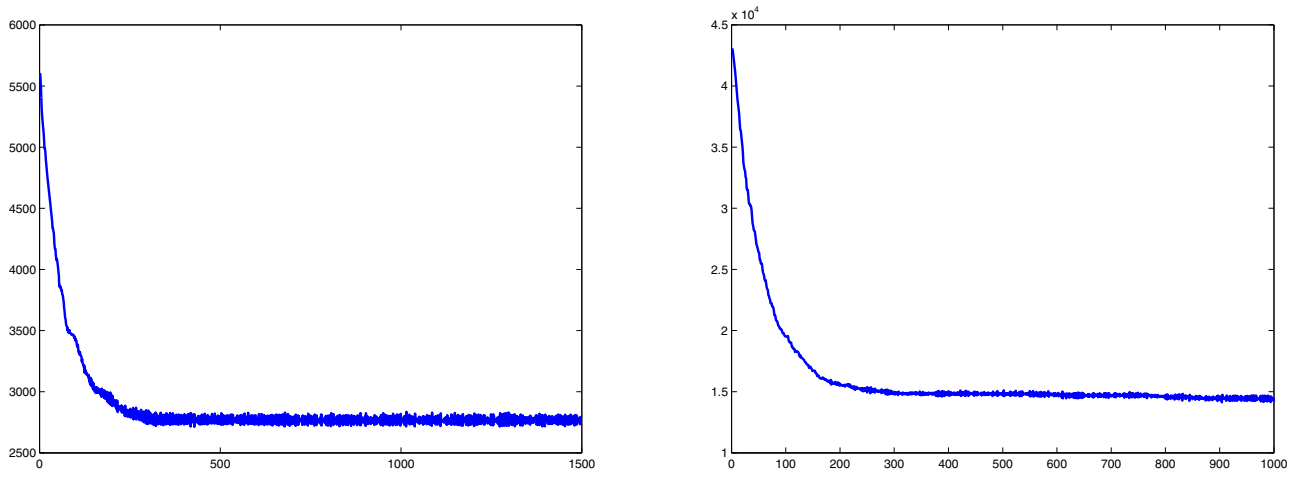

Fig. 19. Singular case: $T=1, a=10$. Energy $E\left(\omega_{k}, a, T=1\right)$ with respect to $k$ without (left) and with (right) viscosity terms.

we observe that the modified scheme leads to a significantly lower energy (see Fig 19). Without viscosity terms, we obtain $E(\omega, a, T) \approx 2768.70$, whereas with viscosity terms, we obtain $E(\omega, a, T) \approx 1487.23$. Once again, in spite of the symmetry of $y_{0}$, the centered disc $\omega_{(1 / 2)}$ of area $L$ is not the optimal domain: we compute $E\left(\omega_{(1 / 2)}, a, T\right) \approx 1984.72$.

\section{Concluding remarks}

We have numerically solved the shape design problem, which consists in optimizing the support of a damped term for the linear wave equation. This work completes the previous theoretical study performed in (Münch et al., 2006), where a well-posed relaxation for $\left(P_{\omega}\right)$ is derived. In agreement with (Münch et al., 2006), the numerical experiments highlights the crucial influence of the overdamping phenomenon on the optimal position. On the one hand, when the damping coefficient is small enough (this depends on the data of the problem), Problem $\left(P_{\omega}\right)$ is well posed: the topological derivative then provides a good approximation of the design, improved iteratively by the level set method. On the other hand, when the damping coefficient is large, the problem is ill posed and the algorithm yields local optima. First, this illustrates the complexity and richness of the problem in contrast to the apparent simplicity of the linear system. Secondly, it also emphasizes the efficiency of this descent method coupled with a level set approach to detect or not local minima. Moreover, when the support is fixed, the dissipation is optimized with a high gradient and a locally negative damping function. Once again, this result, in agreement with the literature, is due to the over-damping phenomenon.

The case of boundary dissipation may be numerically analyzed in a similar way as others models such as piezo-elastic systems (Degryse and Mottelet, 2005). For exact controllability of the wave equation, we refer to (Münch, 2008), where the optimal shape of the control is analyzed. Finally, adapting (Maestre et al., 2007) and
(Zolésio and Truchi, 1988), it seems interesting and challenging to analyze the non-cylindrical situation where the support $\omega$ may depend on time.

\section{Acknowledgment}

The work was partially supported by the grants ANR-05JC-0182-01 and ANR-07-JC-1832-84.

\section{References}

Allaire, G., de Gournay, F., Jouve, F. and Toader, A. (2005) Structural optimization using topological and shape sensitivity analysis via a level-set method, Control and Cybernetics 34(1): 59-80.

Allaire, G., Jouve, F. and Toader, A. (2004). Structural optimization using sensitivity analysis and a level-set method, Journal of Computational Physics 194(1): 363-393.

Banks, H., Ito, K. and Wang, B. (1991). Exponentially stable approximations of weakly damped wave equations, Estimation and Control of Distributed Parameter Systems (Vorau, 1990), International Series of Numerical Mathematics, Vol. 100, Birkhäuser, Basel, pp. 1-33.

Bardos, C., Lebeau, G. and Rauch, J. (1992). Sharp sufficient conditions for the observation, control and stabilization from the boundary, SIAM Journal on Control and Optimization 30(5): 1024-1065.

Burger, M. and Osher, S. (2005). A survey on level set methods for inverse problems and optimal design, European Journal of Applied Mathematics 16(2): 263-301.

Cagnol, J. and Zolésio, J.-P. (1999). Shape derivative in the wave equation with Dirichlet boundary conditions, Journal of Differential Equations 158(2): 175-210.

Castro, C. and Cox, S.J. (2001). Achieving arbitrarily large decay in the damped wave equation, SIAM Journal on Control and Optimization 39(6): 1748-1755.

Cohen, G.C. (2002). Higher-order Numerical Methods for Transient Wave Equations, Scientific Computation, SpringerVerlag, Berlin. 
Degryse, E. and Mottelet, S. (2005). Shape optimization of piezoelectric sensors or actuators for the control of plates, ESAIM Control, Optimization and Calculus of Variations 11(4): 673-690.

Delfour, M. and Zolesio, J. (2001). Shapes and GeometriesAnalysis, Differential Calculus and Optimization, SIAM, Philadelphia, PA.

Fahroo, F. and Ito, K. (1997). Variational formulation of optimal damping designs, Optimization Methods in Partial Differiential Equations (South Hadley, MA, 1996), Contemporary Mathematics, Vol. 209, American Mathematical Society, Providence, RI, pp. 95-114.

Freitas, P. (1999). Optimizing the rate of decay of solutions of the wave equation using genetic algorithms: A counterexample to the constant damping conjecture, SIAM Journal on Control and Optimization 37(2): 376-387.

Fulmanski, P., Laurain, A., Scheid, J.-F. and Sokołowski, J. (2008). Level set method with topological derivatives in shape optimization, International Journal of Computer Mathematics 85(10): 1491-1514.

Glowinski, R., Kinton, W. and Wheeler, M. (1989). A mixed finite element formulation for the boundary controllability of the wave equation, International Journal for Numerical Methods in Engineering 27(3): 623-635.

Hébrard, P. and Henrot, A. (2003). Optimal shape and position of the actuators for the stabilization of a string. Optimization and control of distributed systems Systems and Control Letters 48(3-4): 199-209.

Hébrard, P. and Henrot, A. (2005). A spillover phenomenon in the optimal location of actuators, SIAM Journal on Control and Optimization 44(1): 349-366.

Henrot, A. and Pierre, M. (2005). Variation et optimisation de formes-Une analyse géométrique, Mathématiques et Applications, Vol. 48, Springer, Berlin.

Lions, J. and Magenes, E. (1968). Problèmes aux Limites Non Homogènes et Applications, Dunod, Paris.

López-Gómez, J. (1997). On the linear damped wave equation, Journal of Differential Equations 134(1): 26-45.

Maestre, F., Münch, A. and Pedregal, P. (2007). A spatiotemporal design problem for a damped wave equation, SIAM Journal on Applied Mathematics 68(1): 109-132.

Münch, A. (2008). Optimal design of the support of the control for the 2-d wave equation: Numerical investigations, Mathematical Modelling and Numerical Analysis 5(2): 331351.

Münch, A. and Pazoto, A. (2007). Uniform stabilization of a numerical approximation of the locally damped wave equation, Control, Optimization and Calculus of Variations 13(2): 265-293.

Münch, A., Pedregal, P. and Periago, F. (2006). Optimal design of the damping set for the stabilization of the wave equation, Journal of Differential Equations 231(1): 331-358.

Münch, A., Pedregal, P. and Periago, F. (2009). Optimal internal stabilization of the linear system of elasticity, Archive Rational Mechanical Analysis, (to appear), DOI: 10.1007/s00205-008-0187-4.
Osher, S. and Fedkiw, R. (1996). Level Set Methods: Evolving Interfaces in Geometry, Fluid Mechanics, Computer Vision, and Materials Science, Cambridge University Press, Cambridge.

Osher, S. and Fedkiw, R. (2003). Level Set Methods and Dynamic Implicit Surfaces, Applied Mathematical Sciences, Vol. 153, Springer-Verlag, New York, NY.

Osher, S. and Sethian, J.A. (1988). Fronts propagating with curvature-dependent speed: Algorithms based on Hamilton-Jacobi formulations, Journal of Computational Physics 79(1): 12-49.

Sokołowski, J. and Żochowski, A. (1999). On the topological derivative in shape optimization, SIAM Journal on Control and Optimization 37(4): 1251-1272.

Wang, M.Y., Wang, X. and Guo, D. (2003). A level set method for structural topology optimization, Computer Methods in Applied Mechanics and Engineering 192(1-2): 227-246.

Zolésio, J.-P. and Truchi, C. (1988). Shape stabilization of wave equation, Boundary Control and Boundary Variations (Nice, 1986), Lectures Notes in Computer Science, Vol. 100, Springer, Berlin, pp. 372-398.

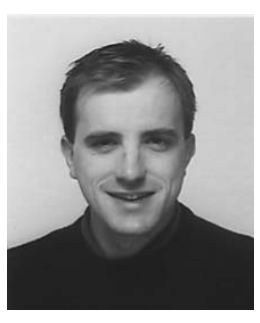

Arnaud Münch was born in France in 1974 He obtained his Ph.D. in applied mathematics in 2002 at the University Paris 6, France. The subject was concerned with the propagation of cracks in shell structures as well as the analysis of nonlinear bonded joint models. He then studied the control theory of dynamical systems, with a one-year stay in Madrid in a group leaded by Enrique Zuazua. Arnaud Münch is now an assistant professor at the Department of Mathematics of Franche-Comté, France, and studies in particular the control of elastic structures as well as optimal shape design problems related to time dependent systems. He has recently received his habilitation à diriger des recherches.

Received: 11 February 2008 Revised: 7 August 2008 
